\title{
A New Mixed Basis Navier-Stokes Formulation for Incompressible Flows over Complex Geometries
}

\author{
Avinaash Murali*, R.G. Rajagopalan \\ Department of Aerospace Engineering, Iowa State University, Ames, Iowa, 50011, USA
}

\begin{abstract}
Numerical modeling of complex geometries necessitates the use of curvilinear body fitted coordinates. This article proposes a novel mixed basis formulation of the governing conservation equations for general curvilinear non-orthogonal grids with the physical covariant velocity as the primary solution variable. This results in an algorithm which has many advantages of orthogonal equations. The conservation equations written in this form retains the diagonal dominance of the pressure equation. The newly formed conservation equations are solved on a structured grid using the SIMPLER algorithm and are shown to converge well for non-orthogonal grids. Standard $\mathcal{K}-\epsilon$ model is used for the turbulence closure.
\end{abstract}

Keywords: Mixed formulation, Navier - Stokes equations, Complex geometry

\section{Introduction}

Fluid flows over complex geometries can be numerically analyzed using general curvilinear non-orthogonal Body Fitted Coordinates (BFC). The motivation for the present research is to develop the governing conservation equations suitable for convection dominated, incompressible flows over complex geometry using BFC. A coordinate invariant vector can be decomposed into different components like Cartesian, contravariant or covariant in a general non-orthogonal system. Researchers have developed several formulations with different choice of velocity components as the primary solution variable. Each formulation has its own advantages and disadvantages. The most straightforward approach is to use the Cartesian velocity components as the primary unknown variables. Rhie and Chow [1], Lien and Leschziner [2] implemented this formulation in a collocated grid whereas Shyy et al. [3] implemented it in a staggered grid. The use of Cartesian velocity components always results in momentum equations with multi-directional pressure gradient terms irrespective of orthogonal or non-orthogonal grids (with the exception of the trivial case of a Cartesian grid system). The usual practice is to ignore the non-orthogonality while deriving the pressure and pressure-correction equation. Peric [4] noted that as the deviation from orthogonal grid becomes severe, dropping non-orthogonal terms results in failure of convergence. To overcome the convergence problem, non-directional pressure gradient terms need to be considered while deriving the pressure equation. Consequently, the resulting stencil of the discretized pressure equation involves diagonal direction grid points in addition to the neighboring grid points. Apart from giving rise to more terms and a complex stencil, the non-orthogonality results in loss of diagonal dominance in the discretized pressure equation matrix. Iterative solvers while operating on such matrices fail to converge, especially when the grid non-orthogonality is severe [4].

\footnotetext{
* Corresponding author

Email addresses: avinaash@iastate.edu (Avinaash Murali), rajagopa@iastate.edu (R.G. Rajagopalan)
} 
In the context of pressure equation, the grid orientation can be leveraged to our advantage by using curvilinear velocity components. Many researchers have used either contravariant or covariant velocity components and have come up with different forms of the governing equation. When curvilinear velocity components are used, the spatially varying nature of the basis vector gives rise to additional flux and source terms in the governing equation. Convective and diffusive terms contain secondary non-directional fluxes that need to be treated explicitly as part of the source term. In high Reynolds number flows the convection terms dominate over the diffusion terms. In the pressure based solution procedures of incompressible flow it is desirable to treat convection terms implicitly while secondary diffusive fluxes may still be treated explicitly. Demirdzic et al. [5], Yang et al. [6], and Graef et al. [7] used physical contravariant velocity for flow calculation in complex geometries. Although such a formulation represents the convective fluxes compactly and enables their implicit treatment with ease, the lack of diagonal dominance of the pressure equation still persist due to the presence of multi-directional pressure gradient in the momentum equations. Sharatchandra and Rhode [8] followed a vector discretization procedure for steady flows with the physical contravariant velocity components as the primary variable. The final discrete equations contain cross-directional pressure gradient terms indirectly. When their solution method is applied to unsteady flows, each discretized momentum equation will have additional time derivative terms of the non-directional velocity components that need to be treated explicitly. They presented test cases that have moderate grid non-orthogonality. Karki and Patankar [9] and Tamamidis and Assanis [10] demonstrated that by using covariant velocity projections as the primary solution variable, governing equations with unidirectional pressure gradient term might be obtained. However, the divergence of convection-diffusion flux tensor expressed in pure covariant components yields far too many terms. The convective flux across a face cannot be represented with a single velocity component alone and contains secondary non-directional convective fluxes. Karki and Patankar [9] avoided the use of tensor transformation and the discrete equations for covariant velocity projection was obtained by algebraic manipulation of the discrete equations for Cartesian components. The mathematics of Karki's method for discretization is simple and yields a straight forward method for the numerical algorithm using covariant velocities. However, the resulting equations are useful in the discretized form only and are not amenable to analytic simplifications.

The foregoing discussion leads to the motivation of the present research - a pressure based solution procedure for incompressible flow using governing equations which has unidirectional pressure gradient and compact representation of the convective flux. In this article, the governing conservation equations are redeveloped by expressing the flux tensor in a new mixed contravariant-covariant basis with physical covariant velocities as the primary solution variable. It is demonstrated that by doing so, the face flux can be compactly represented while still retaining the diagonal dominance of the pressure equation. It must be stressed here that the way physical velocity components are defined and used in this paper is different from $[7,9,10]$. The present definition is consistent with the convention adopted in the tensor theory and by many other researchers $[11,12]$. The equations developed in the mixed basis for the general non-orthogonal coordinates closely resemble the orthogonal equations. As a result, an existing orthogonal solver can be extended to a non-orthogonal solver with minimal changes. The proposed procedure is generic, containing complex secondary source terms and could be used to study a variety of problems. However, many practical engineering problems can be solved without the necessity for a completely generic and complex grid. Even in a problem that has complex geometry, the grids in the entire domain need not be curvilinear. Quite often, grids are non-orthogonal in the vicinity of a body. Far away, they can be orthogonal and in most cases even Cartesian. Since the present governing equations are obtained in a differential form, the secondary source terms can be evaluated mathematically and simplified for different grids. The Reynolds Averaged Navier-Stokes (RANS) equations are used to solve the flow-field using SIMPLER algorithm of Patankar [13] and the Standard $\mathcal{K}-\epsilon$ model of Launder and Spalding [14] is used for turbulence closure.

\section{Mathematical convention}

In this section, we briefly establish the mathematical convention adopted in this article. In a curvilinear coordinate system $x^{i}$, with $\boldsymbol{R}$ representing a general position vector, the mutually reciprocal covariant $\boldsymbol{e}_{\boldsymbol{i}}$ 
and contravariant $\boldsymbol{e}^{\boldsymbol{i}}$ basis vectors are respectively defined as

$$
\begin{aligned}
\boldsymbol{e}_{\boldsymbol{i}} & =\frac{\partial \boldsymbol{R}}{\partial x^{i}} \\
\boldsymbol{e}^{\boldsymbol{i}} & =\nabla x^{i}=\frac{1}{2 \sqrt{g}} \epsilon^{i j k}\left(\boldsymbol{e}_{\boldsymbol{j}} \times \boldsymbol{e}_{\boldsymbol{k}}\right)
\end{aligned}
$$

Here $\epsilon^{i j k}$ denotes the Levi-Civita operator. The respective unit basis vectors $\hat{\boldsymbol{e}}_{\boldsymbol{i}}$ and $\hat{\boldsymbol{e}}^{\boldsymbol{i}}$ are obtained by normalizing the basis vectors with their corresponding scale factors $h_{i}$ and $h^{i}$. Throughout this paper, no summation is implied on the repeated indices when used with scale factors.

$$
\begin{array}{ll}
\hat{\boldsymbol{e}}_{\boldsymbol{i}}=\frac{\boldsymbol{e}_{\boldsymbol{i}}}{h_{i}} & h_{i}=\left|\boldsymbol{e}_{\boldsymbol{i}}\right| \\
\hat{\boldsymbol{e}}^{\boldsymbol{i}}=\frac{\boldsymbol{e}^{\boldsymbol{i}}}{h^{i}} & h^{i}=\left|\boldsymbol{e}^{\boldsymbol{i}}\right|
\end{array}
$$

The covariant $g_{i j}$ and contravariant $g^{i j}$ metric tensors are given by,

$$
\begin{aligned}
\boldsymbol{e}_{\boldsymbol{i}} \cdot \boldsymbol{e}_{\boldsymbol{j}} & =g_{i j} \\
\boldsymbol{e}^{\boldsymbol{i}} \cdot \boldsymbol{e}^{\boldsymbol{j}} & =g^{i j}
\end{aligned}
$$

In general coordinates, a vector can be described using contravariant components $V^{i}$ along covariant basis or covariant components $V_{i}$ along contravariant basis.

$$
\boldsymbol{V}=V^{i} e_{i}=V_{i} e^{i}
$$

Using the covariant and contravariant metric tensors, the components can be interchanged.

$$
\begin{aligned}
V_{i} & =g_{i j} V^{j} \\
V^{i} & =g^{i j} V_{j}
\end{aligned}
$$

The square root of the determinant $\sqrt{g}$, of the covariant metric tensor is also equal to the determinant of the inverse Jacobian transformation matrix.

$$
\sqrt{g}=\sqrt{\operatorname{det}\left(g_{i j}\right)}=\left|\frac{\partial(x, y, z)}{\partial\left(x^{1}, x^{2}, x^{3}\right)}\right|
$$

Differentiation of the basis vectors give rise to metrics called Christoffel symbols of the second kind and they are given by,

$$
\Gamma_{i j}^{k}=\Gamma_{j i}^{k}=\frac{\partial e_{i}}{\partial x^{j}} \cdot e^{k}
$$

\section{New mixed basis formulation}

In orthogonal curvilinear coordinates, the convective flux is evaluated using single velocity component and the momentum equations contain unidirectional pressure gradient. Similar form of momentum equations in non-orthogonal curvilinear coordinates can be obtained by adopting a mixed basis representation for the flux tensor. The convective-diffusive fluxes appearing in the momentum equations are second rank tensors. The convective flux consists of a dyadic product of the velocity vector with itself and the diffusive flux contains gradient of the velocity vector. The conventional approach adopted in literature is to represent this flux tensor $\tilde{\boldsymbol{T}}$, using either pure covariant $T_{i j} \boldsymbol{e}^{\boldsymbol{i}} \boldsymbol{e}^{j}$ or contravariant $T^{i j} \boldsymbol{e}_{\boldsymbol{i}} \boldsymbol{e}_{\boldsymbol{j}}$ components. In the present research, this tensor is expressed using mixed components in the contravariant-covariant basis $T_{i}^{j} \boldsymbol{e}^{i} \boldsymbol{e}_{j}$. By expressing the flux tensor in a mixed basis as shown in this paper, the advantages of both the contravariant and covariant formulations can be realized. Figure 1 shows the direction associated with the contravariant and covariant velocity components. 


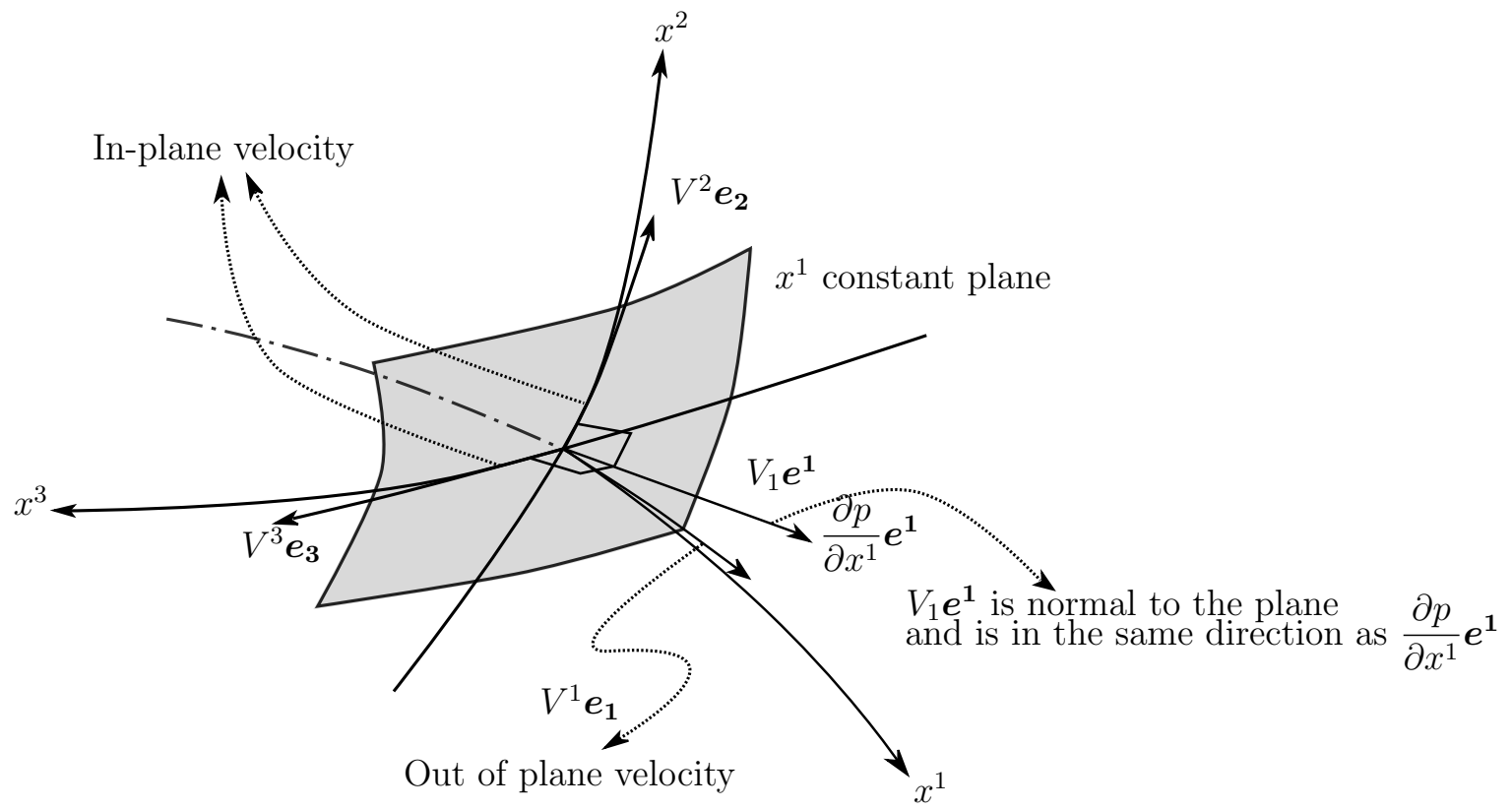

Fig. 1. Representation of the convective flux dyad as a mixed tensor. The vectors in this figure imply only the direction and not the magnitude.

Upon applying the Gauss-divergence theorem and integrating over a finite control volume, the second $\boldsymbol{V}$ of the dyad $\rho \boldsymbol{V} \boldsymbol{V}$ dots with elemental area $\boldsymbol{d} \boldsymbol{A}$ to give the mass flux across a face. This $\boldsymbol{V}$ shall be represented using the contravariant components. On any arbitrary plane in a three-dimensional space, two of the three contravariant components would lie on the plane and only a single contravariant component would contribute to the mass flux entering or leaving the face. The choice of contravariant component for the second $\boldsymbol{V}$ of the dyad ensures that there is no secondary convective flux.

To represent the primary solution variable along the direction of the pressure gradient, the first $\boldsymbol{V}$ of the dyad $\rho \boldsymbol{V} \boldsymbol{V}$ is expressed in covariant components. This ensures unidirectional pressure gradient term in the momentum equations, leading to diagonal dominance and compact stencil in the discretized pressure equation. By using such a mixed contravariant-covariant form for the flux tensor in a non-orthogonal basis, treatment of the convective flux and the pressure differential are made similar to an orthogonal basis.

\section{Governing equations}

\subsection{Reynolds Averaged Navier-Stokes equations}

The Reynolds Averaged Navier-Stokes (RANS) equations with Boussinesq's eddy viscosity hypothesis for the Reynolds stress are used to model the incompressible, turbulent flow. The mass and momentum conservation can be written in a coordinate independent form as,

$$
\begin{aligned}
\frac{\partial \rho}{\partial t}+\boldsymbol{\nabla} \cdot(\rho \boldsymbol{V}) & =0 \\
\frac{\partial \rho \boldsymbol{V}}{\partial t}+\boldsymbol{\nabla} \cdot \tilde{\boldsymbol{T}} & =-\boldsymbol{\nabla} \cdot(p \tilde{\boldsymbol{I}})-\boldsymbol{\nabla} \cdot\left(\frac{2}{3} \rho \mathcal{K} \tilde{\boldsymbol{I}}\right)
\end{aligned}
$$

The convection-diffusion flux tensor $\tilde{\boldsymbol{T}}$ is

$$
\tilde{\boldsymbol{T}}=\rho \boldsymbol{V} \boldsymbol{V}-\left[\left(\mu+\mu_{t}\right)\left(\boldsymbol{\nabla} \boldsymbol{V}+\boldsymbol{\nabla} \boldsymbol{V}^{\boldsymbol{T}}\right)\right]
$$

where $\rho, p, \boldsymbol{V}, \mu$ and $\mu_{t}$ are the density, mean pressure, mean velocity vector, molecular and turbulent eddy viscosity respectively. To model the turbulence, two equation standard $\mathcal{K}-\epsilon$ model with Launder and 
Spalding [14] wall function is used. The transport equations for the turbulent kinetic energy $\mathcal{K}$ and the turbulent dissipation rate $\epsilon$ are

$$
\begin{aligned}
\frac{\partial \rho \mathcal{K}}{\partial t}+\nabla \cdot(\rho \boldsymbol{V} \mathcal{K}) & =\boldsymbol{\nabla} \cdot\left[\left(\mu+\frac{\mu_{t}}{\sigma_{\mathcal{K}}}\right) \nabla \mathcal{K}\right]+P_{\mathcal{K}}-\rho \epsilon \\
\frac{\partial \rho \epsilon}{\partial t}+\nabla \cdot(\rho \boldsymbol{V} \epsilon) & =\boldsymbol{\nabla} \cdot\left[\left(\mu+\frac{\mu_{t}}{\sigma_{\epsilon}}\right) \nabla \epsilon\right]+C_{1 \epsilon} \frac{\epsilon}{\mathcal{K}} P_{\mathcal{K}}-C_{2 \epsilon} \rho \frac{\epsilon^{2}}{\mathcal{K}}
\end{aligned}
$$

Here $P_{\mathcal{K}}$ and $\rho \epsilon$ are the production and dissipation of the turbulent kinetic energy. The production term is modeled along the lines of Kato [15]. $P_{\mathcal{K}}$ can be related to the modulus $S$ of the mean strain rate tensor $\tilde{\boldsymbol{S}}$, and the modulus $\Omega$ of the mean rotation rate tensor $\tilde{\Omega}$, as $P_{\mathcal{K}}=\mu_{t} S \Omega$. The symmetric and anti-symmetric parts of the velocity gradient give the mean strain rate and the mean rotation rate tensors.

$$
\begin{aligned}
\tilde{\boldsymbol{S}} & =\frac{1}{2}\left[\nabla \boldsymbol{V}+\boldsymbol{\nabla} \boldsymbol{V}^{\boldsymbol{T}}\right] \\
\tilde{\boldsymbol{\Omega}} & =\frac{1}{2}\left[\nabla \boldsymbol{V}-\boldsymbol{\nabla} \boldsymbol{V}^{\boldsymbol{T}}\right]
\end{aligned}
$$

Their respective moduli are defined as $S=\sqrt{2 \tilde{\boldsymbol{S}}: \tilde{\boldsymbol{S}}}$ and $\Omega=\sqrt{2 \tilde{\boldsymbol{\Omega}}: \tilde{\boldsymbol{\Omega}}}$. The operator $:$ refers to the double dot product between two tensors [12]. The turbulent eddy viscosity is then given by $\mu_{t}=C_{\mu} \rho \mathcal{K}^{2} / \epsilon$. The standard model constants are $\sigma_{\mathcal{K}}=1.0, \sigma_{\epsilon}=1.3, C_{\mu}=0.09, C_{1 \epsilon}=1.44$, and $C_{2 \epsilon}=1.92$.

\subsection{Governing equations in curvilinear coordinates with mixed components}

The preceding set of coordinate invariant governing equations is expanded in the curvilinear coordinates $x^{i}$ with covariant velocity (along the contravariant basis) as the primary unknown quantity. To do so, the following vector and tensor operations are necessary.

1. Using covariant components, the divergence of a vector $\left(\boldsymbol{V}=V_{j} \boldsymbol{e}^{\boldsymbol{j}}\right)$ is

$$
\boldsymbol{\nabla} \cdot \boldsymbol{V}=\frac{1}{\sqrt{g}} \frac{\partial}{\partial x^{i}}\left(\sqrt{g} g^{i j} V_{j}\right)
$$

2. The gradient of a scalar $\phi$

$$
\boldsymbol{\nabla} \phi=\nabla \cdot(\phi \tilde{\boldsymbol{I}})=\frac{\partial \phi}{\partial x^{i}} e^{i}
$$

3. The divergence of a second rank tensor $\left(\tilde{\boldsymbol{T}}=T_{i}^{j} \boldsymbol{e}^{\boldsymbol{i}} \boldsymbol{e}_{\boldsymbol{j}}\right)$ using mixed components

$$
\boldsymbol{\nabla} \cdot \tilde{\boldsymbol{T}}=\left[\frac{1}{\sqrt{g}} \frac{\partial}{\partial x^{j}}\left(\sqrt{g} T_{i}^{j}\right)-\Gamma_{i j}^{k} T_{k}^{j}\right] \boldsymbol{e}^{i}
$$

For the proofs of Eqs. (19), (20) Warsi [12] may be referred. The divergence of a second rank tensor in mixed components of Eq. (21) is explained in the appendix Eq. (A.10). Mass conservation of Eq. (12) is written using Eq. (19), as

$$
\frac{\partial \rho}{\partial t}+\frac{1}{\sqrt{g}} \frac{\partial}{\partial x^{i}}\left(\rho \sqrt{g} g^{i j} V_{j}\right)=0
$$

The momentum conservation of Eq. (13) is expanded using Eqs. (20), (21) as,

$$
\frac{\partial \rho V_{i}}{\partial t}+\frac{1}{\sqrt{g}} \frac{\partial}{\partial x^{j}}\left(\sqrt{g} T_{i}^{j}\right)=-\frac{\partial p}{\partial x^{i}}-\frac{\partial}{\partial x^{i}}\left(\frac{2}{3} \rho \mathcal{K}\right)+\Gamma_{i j}^{k} T_{k}^{j}
$$

Equations (22), (23) are expressed using non-physical components $V_{j}$ and $T_{i}^{j}$ that are defined with respect to non-normalized bases $\boldsymbol{e}^{j}$ and $\boldsymbol{e}^{\boldsymbol{i}} \boldsymbol{e}_{\boldsymbol{j}}$. These non-physical components have scale factors associated 
with them and do not have the same dimensions as the physical quantities. Yang et al. [11] noted that the non-physical components exhibit undesirable mesh sensitivity. Therefore, the non-physical components $V_{j}$ and $T_{i}^{j}$ can be replaced with their corresponding physical counterparts $v_{j}$ and $t_{i}^{j}$ (defined with respect to unit bases $\hat{e}^{j}$ and $\hat{e}^{i} \hat{e}_{j}$ ) using

$$
\begin{aligned}
V_{j} & =\frac{v_{j}}{h^{j}} \\
T_{i}^{j} & =\frac{t_{i}^{j}}{h^{i} h_{j}}
\end{aligned}
$$

Using Eqs. (24), (25), after some rearrangement, Eqs. (22), (23), can be rewritten as follows.

$$
\begin{gathered}
\frac{\partial \rho}{\partial t}+\frac{1}{\sqrt{g}} \frac{\partial}{\partial x^{i}}\left(\frac{\rho \sqrt{g} g^{i j} v_{j}}{h^{j}}\right)=0 \\
\frac{\partial \rho v_{i}}{\partial t}+\frac{1}{\sqrt{g}} \frac{\partial}{\partial x^{j}}\left(\frac{\sqrt{g} t_{i}^{j}}{h_{j}}\right)=-h^{i} \frac{\partial p}{\partial x^{i}}-h^{i} \frac{\partial}{\partial x^{i}}\left(\frac{2}{3} \rho \mathcal{K}\right)+\frac{\Gamma_{i j}^{k} h^{i} t_{k}^{j}}{h^{k} h_{j}}+\frac{\partial h^{i}}{\partial x^{j}} \frac{t_{i}^{j}}{h^{i} h_{j}}
\end{gathered}
$$

Employing the Kronecker delta function to change the index of the final term in the RHS from $i$ to $k$, the above equation is rewritten in a more compact form.

$$
\frac{\partial \rho v_{i}}{\partial t}+\frac{1}{\sqrt{g}} \frac{\partial}{\partial x^{j}}\left(\frac{\sqrt{g} t_{i}^{j}}{h_{j}}\right)=-h^{i} \frac{\partial p}{\partial x^{i}}-h^{i} \frac{\partial}{\partial x^{i}}\left(\frac{2}{3} \rho \mathcal{K}\right)+\left(\Gamma_{i j}^{k} h^{i}+\delta_{i k} \frac{\partial h^{k}}{\partial x^{j}}\right) \frac{t_{k}^{j}}{h^{k} h_{j}}
$$

The contravariant-covariant mixed component representation of the second rank convective flux dyad and the gradient of the velocity vector (see Eq. (B.8)) in the diffusive flux are given by

$$
\begin{aligned}
\rho \boldsymbol{V} \boldsymbol{V} & =\rho v_{i} v^{j} \hat{\boldsymbol{e}}^{i} \hat{\boldsymbol{e}}_{\boldsymbol{j}} \\
\boldsymbol{\nabla} \boldsymbol{V} & =h^{i} h_{j}\left[\frac{\partial}{\partial x^{k}}\left(\frac{v_{i}}{h^{i}}\right)-\frac{v_{l}}{h^{l}} \Gamma_{i k}^{l}\right] g^{k j} \hat{\boldsymbol{e}}^{i} \hat{\boldsymbol{e}}_{\boldsymbol{j}}
\end{aligned}
$$

The contravariant velocity $v^{j}$, is calculated from the covariant velocity $v_{i}$, using Eqs. (9), (24). The convective flux for $\mathcal{K}$ equation can be expanded using Eqs. (19), (9) and replaced with physical components similar to Eq. (24).

$$
\nabla \cdot(\rho \boldsymbol{V} \mathcal{K})=\frac{1}{\sqrt{g}} \frac{\partial}{\partial x^{i}}\left(\rho \sqrt{g} g^{i j} V_{j} \mathcal{K}\right)=\frac{1}{\sqrt{g}} \frac{\partial}{\partial x^{i}}\left(\rho \sqrt{g} V^{i} \mathcal{K}\right)=\frac{1}{\sqrt{g}} \frac{\partial}{\partial x^{i}}\left(\frac{\rho \sqrt{g} v^{i} \mathcal{K}}{h_{i}}\right)
$$

The diffusive flux for $\mathcal{K}$ equation can be expanded using Eqs. (19), (20).

$$
\nabla \cdot\left[\left(\mu+\frac{\mu_{t}}{\sigma_{\mathcal{K}}}\right) \nabla \mathcal{K}\right]=\frac{1}{\sqrt{g}} \frac{\partial}{\partial x^{i}}\left[\left(\mu+\frac{\mu_{t}}{\sigma_{\mathcal{K}}}\right) \sqrt{g} g^{i j} \frac{\partial \mathcal{K}}{\partial x^{j}}\right]
$$

Therefore, the complete transport equation for turbulent kinetic energy $\mathcal{K}$ is,

$$
\frac{\partial \rho \mathcal{K}}{\partial t}+\frac{1}{\sqrt{g}} \frac{\partial}{\partial x^{i}}\left(\frac{\rho \sqrt{g} v^{i} \mathcal{K}}{h_{i}}\right)=\frac{1}{\sqrt{g}} \frac{\partial}{\partial x^{i}}\left[\left(\mu+\frac{\mu_{t}}{\sigma_{\mathcal{K}}}\right) \sqrt{g} g^{i j} \frac{\partial \mathcal{K}}{\partial x^{j}}\right]+P_{\mathcal{K}}-\rho \epsilon
$$

The transport equation for the turbulent dissipation rate $\epsilon$ is obtained in a similar manner.

$$
\frac{\partial \rho \epsilon}{\partial t}+\frac{1}{\sqrt{g}} \frac{\partial}{\partial x^{i}}\left(\frac{\rho \sqrt{g} v^{i} \epsilon}{h_{i}}\right)=\frac{1}{\sqrt{g}} \frac{\partial}{\partial x^{i}}\left[\left(\mu+\frac{\mu_{t}}{\sigma_{\epsilon}}\right) \sqrt{g} g^{i j} \frac{\partial \epsilon}{\partial x^{j}}\right]+C_{1 \epsilon} \frac{\epsilon}{\mathcal{K}} P_{\mathcal{K}}-C_{2 \epsilon} \rho \frac{\epsilon^{2}}{\mathcal{K}}
$$


The modulus of a tensor is needed in the production term and is evaluated with the double dot product. Substituting for $\boldsymbol{\nabla} \boldsymbol{V}$ from Eq. (30), the mean strain rate tensor of Eq. (17) can be written in the form $\tilde{\boldsymbol{S}}=s_{i}^{j} \hat{\boldsymbol{e}}^{i} \hat{\boldsymbol{e}}_{\boldsymbol{j}}$. The double dot product is then evaluated as

$$
\tilde{\boldsymbol{S}}: \tilde{\boldsymbol{S}}=s_{i}^{j} \hat{\boldsymbol{e}}^{i} \hat{\boldsymbol{e}}_{\boldsymbol{j}}: s_{m}^{n} \hat{\boldsymbol{e}}^{\boldsymbol{m}} \hat{\boldsymbol{e}}_{\boldsymbol{n}}=\frac{s_{i}^{j}}{h^{i} h_{j}} \frac{s_{m}^{n}}{h^{m} h_{n}} g^{i m} g_{j n}
$$

The modulus of the mean rotation rate tensor is evaluated similarly and the production term is calculated through $P_{\mathcal{K}}=\mu_{t} S \Omega$. The momentum equation expressed using mixed components has the following advantages.

1. The convective flux is represented compactly and the mass flow rate across the face of a control volume can be expressed using a single contravariant velocity component alone. The convection has no secondary flux. With formulations that do not use contravariant velocity for fluxes, secondary non-directional fluxes arise. In order to treat them in an implicit manner, all the components of the velocities need to be present on every face of the control volume, thus requiring interpolation of the non-directional velocities onto all faces.

2. The choice of physical covariant velocity as the primary solution variable has resulted in unidirectional pressure gradient term in the momentum equation. This is true irrespective of whether the grids are orthogonal or non-orthogonal. In the discretized equation, the pressure at each grid point is related only to the neighboring grid points along the coordinate direction, leading to a simple 5 point stencil in $2 \mathrm{D}$ or 7 point stencil in 3D. The resulting matrix of the discretized pressure equation will be diagonally dominant (see Section 5.3) and aids convergence in an iterative solution procedure. In contrast, if multi-directional pressure gradient terms are present, the discrete pressure equation will require a complex 9 point stencil in $2 \mathrm{D}$ or 21 point stencil in $3 \mathrm{D}$ and lack diagonal dominance. As the incompressible, pressure based, finite volume procedure solves for pressure and pressure correction equation in every iteration, the present formulation is favorable. The identity tensor $\tilde{\boldsymbol{I}}$ in a mixed basis, contains only unit diagonal terms unlike the covariant or contravariant representation of the identity tensor. Thus, the pressure tensor in non-orthogonal coordinates retains a structure similar to the orthogonal coordinates.

$$
p \tilde{\boldsymbol{I}}=p \delta_{i}^{j} \boldsymbol{e}^{i} \boldsymbol{e}_{\boldsymbol{j}}=p g_{i j} \boldsymbol{e}^{i} \boldsymbol{e}^{\boldsymbol{j}}=p g^{i j} \boldsymbol{e}_{\boldsymbol{i}} \boldsymbol{e}_{\boldsymbol{j}}
$$

3. The governing equations are derived for the generic case of a triply non-orthogonal curvilinear grid and are thus applicable to a wide variety of problems. However, all the flow geometries do not necessarily require such a complex grid. Quite often, fluid flow problems can be solved with relatively simpler grids like tubular, rotated, extruded, orthogonal grids and so on. Rather than developing multiple solvers for different grid systems, it would be advantageous to develop a single generic solver that can handle different classes of grids. The momentum equation developed in the differential form is suitable for grid geometry based simplifications. All the terms but one in Eq. (28) are present in every coordinate system. The secondary source term $\left(\Gamma_{i j}^{k} h^{i}+\delta_{i k} \partial h^{k} / \partial x^{j}\right) t_{k}^{j} / h^{k} h_{j}$ alone varies with different coordinate systems. These metrics can be evaluated mathematically and modified correspondingly. This facilitates the development of an efficient solver with optimal use of computer storage and minimal scope for spurious discretization errors.

The close similarity in terms of the equation structure, treatment of convection and pressure in nonorthogonal grid using mixed components to the general orthogonal grid means very little effort is required to extend an orthogonal solver to a non-orthogonal solver.

\section{Finite volume discretization}

A three-dimensional solver based on the proposed formulation has been developed for a structured body conforming grid. Finite volume method is used to discretize and solve the coupled Eqs. (26), (28), (33) 
and (34). A staggered grid has been adopted where $p, \mu, \mu_{t}, \mathcal{K}$, and $\epsilon$ are stored at the cell centers and the velocities are stored at the cell faces to avoid spurious pressure oscillation. A typical control volume around a grid point $P$ is shown in Fig. 2. $E, W, N, S, T$ and $B$ are the neighboring grids points and $e, w, n, s, t$ and $b$ are the corresponding control volume faces.

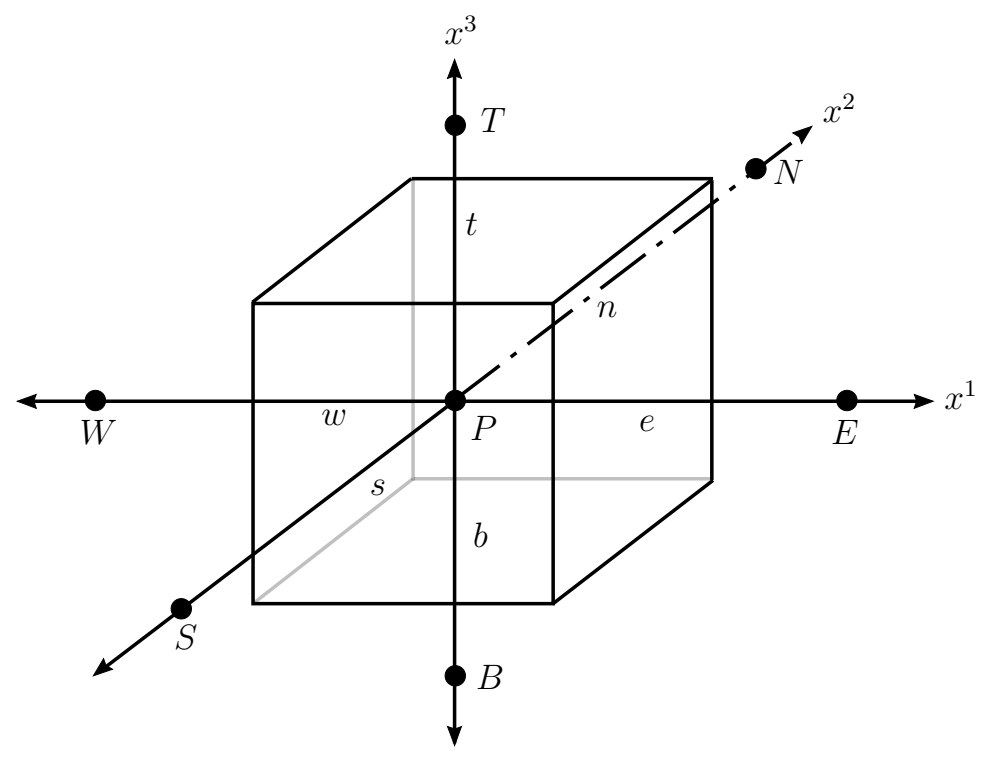

Fig. 2. Control volume around a grid point $P$.

\subsection{Discretization of momentum equation}

Consider the momentum equation in the first direction. Representing the secondary source terms as $S$, the $v_{1}$ momentum equation is

$$
\frac{\partial \rho v_{1}}{\partial t}+\frac{1}{\sqrt{g}} \frac{\partial}{\partial x^{1}}\left(\frac{\sqrt{g} t_{1}^{1}}{h_{1}}\right)+\frac{1}{\sqrt{g}} \frac{\partial}{\partial x^{2}}\left(\frac{\sqrt{g} t_{1}^{2}}{h_{2}}\right)+\frac{1}{\sqrt{g}} \frac{\partial}{\partial x^{3}}\left(\frac{\sqrt{g} t_{1}^{3}}{h_{3}}\right)=-h^{1} \frac{\partial p}{\partial x^{1}}-h^{1} \frac{\partial}{\partial x^{1}}\left(\frac{2}{3} \rho \mathcal{K}\right)+S
$$

The convective-diffusive flux $t_{i}^{j}$, is written as the sum of primary convective-diffusive flux $\phi_{i}^{j}$, and the secondary diffusive flux $\psi_{i}^{j}$. The primary fluxes are

$$
\begin{aligned}
& \phi_{1}^{1}=\rho v_{1} v^{1}-\left(\mu+\mu_{t}\right) h_{1} g^{11} \frac{\partial v_{1}}{\partial x^{1}} \\
& \phi_{1}^{2}=\rho v_{1} v^{2}-\left(\mu+\mu_{t}\right) h_{2} g^{22} \frac{\partial v_{1}}{\partial x^{2}} \\
& \phi_{1}^{3}=\rho v_{1} v^{3}-\left(\mu+\mu_{t}\right) h_{3} g^{33} \frac{\partial v_{1}}{\partial x^{3}}
\end{aligned}
$$

The secondary diffusive fluxes $\psi_{1}^{1}, \psi_{1}^{2}$, and $\psi_{1}^{3}$ are expanded in the appendix (see Eqs. (C.1) - (C.3)) These secondary diffusive terms $\psi$, together with the secondary source term $S$ are treated explicitly.

$$
\begin{aligned}
\frac{\partial \rho v_{1}}{\partial t}+\frac{1}{\sqrt{g}} \frac{\partial}{\partial x^{1}}\left(\frac{\sqrt{g} \phi_{1}^{1}}{h_{1}}\right) & +\frac{1}{\sqrt{g}} \frac{\partial}{\partial x^{2}}\left(\frac{\sqrt{g} \phi_{1}^{2}}{h_{2}}\right)+\frac{1}{\sqrt{g}} \frac{\partial}{\partial x^{3}}\left(\frac{\sqrt{g} \phi_{1}^{3}}{h_{3}}\right)=-h^{1} \frac{\partial p}{\partial x^{1}}-h^{1} \frac{\partial}{\partial x^{1}}\left(\frac{2}{3} \rho \mathcal{K}\right)+S \\
& -\frac{1}{\sqrt{g}} \frac{\partial}{\partial x^{1}}\left(\frac{\sqrt{g} \psi_{1}^{1}}{h_{1}}\right)-\frac{1}{\sqrt{g}} \frac{\partial}{\partial x^{2}}\left(\frac{\sqrt{g} \psi_{1}^{2}}{h_{2}}\right)-\frac{1}{\sqrt{g}} \frac{\partial}{\partial x^{3}}\left(\frac{\sqrt{g} \psi_{1}^{3}}{h_{3}}\right)
\end{aligned}
$$



space

Integrating over a finite control volume $\Delta \forall=\sqrt{g} \Delta x^{1} \Delta x^{2} \Delta x^{3}$ at a grid point $P$, and discretizing in

$$
\begin{aligned}
\frac{\partial\left(\rho v_{1 P}\right)}{\partial t} \Delta \forall & +\left[\frac{\sqrt{g} \Delta x^{2} \Delta x^{3} \phi_{1}^{1}}{h_{1}}\right]_{e}-\left[\frac{\sqrt{g} \Delta x^{2} \Delta x^{3} \phi_{1}^{1}}{h_{1}}\right]_{w}+\left[\frac{\sqrt{g} \Delta x^{1} \Delta x^{3} \phi_{1}^{2}}{h_{2}}\right]_{n}-\left[\frac{\sqrt{g} \Delta x^{1} \Delta x^{3} \phi_{1}^{2}}{h_{2}}\right]_{s} \\
& +\left[\frac{\sqrt{g} \Delta x^{1} \Delta x^{2} \phi_{1}^{3}}{h_{3}}\right]_{t}-\left[\frac{\sqrt{g} \Delta x^{1} \Delta x^{2} \phi_{1}^{3}}{h_{3}}\right]_{b}=-\sqrt{g} h^{1} \Delta x^{2} \Delta x^{3}\left[p_{e}-p_{w}\right]+b_{v_{1 P}}
\end{aligned}
$$

The term $b_{v_{1 P}}$ denotes the sum of integrated turbulent kinetic energy gradient, secondary source terms and secondary diffusive flux terms. Piecewise-linear profile is used to evaluate the derivatives in the Eqs. (38) - (40). Using the symbol $F$, for the strength of convection and $D$, for the diffusion conductance, and defining them as

$$
F_{e}=\left(\frac{\sqrt{g} \Delta x^{2} \Delta x^{3} \rho v^{1}}{h_{1}}\right)_{e} \quad D_{e}=\left(\frac{\sqrt{g} \Delta x^{2} \Delta x^{3}\left(\mu+\mu_{t}\right) g^{11}}{\Delta x^{1}}\right)_{e}
$$

Similar terms are defined at the other faces. The metrics in Eqs. (42), (43) have direct geometric meaning. The area $|\boldsymbol{d} \boldsymbol{A}|$ of $x^{1}$ face is $\left|\Delta x^{2} \boldsymbol{e}_{\mathbf{2}} \times \Delta x^{3} \boldsymbol{e}_{\mathbf{3}}\right|=\left|\sqrt{g} \boldsymbol{e}^{\mathbf{1}} \Delta x^{2} \Delta x^{3}\right|=\left|\sqrt{g} h^{1} \hat{\boldsymbol{e}}^{\mathbf{1}} \Delta x^{2} \Delta x^{3}\right|=\sqrt{g} h^{1} \Delta x^{2} \Delta x^{3}$. The mass flow rate through the $x^{1}$ face is

$$
\begin{aligned}
F=\boldsymbol{d} \boldsymbol{A} \cdot \rho \boldsymbol{V} & =\left(\sqrt{g} \boldsymbol{e}^{\mathbf{1}} \Delta x^{2} \Delta x^{3}\right) \cdot \rho\left(V^{1} \boldsymbol{e}_{\mathbf{1}}+V^{2} \boldsymbol{e}_{\mathbf{2}}+V^{3} \boldsymbol{e}_{\mathbf{3}}\right) \\
& =\sqrt{g} \Delta x^{2} \Delta x^{3} \rho V^{1} \\
& =\frac{\sqrt{g} \Delta x^{2} \Delta x^{3} \rho v^{1}}{h_{1}}
\end{aligned}
$$

The diffusion conductance on the $x^{1}$ face is

$$
\begin{aligned}
D & =\frac{\text { Area of } x^{1} \text { face } \times \text { Diffusion coefficient }}{\text { Normal distance along } x^{1}} \\
& =\frac{\sqrt{g} h^{1} \Delta x^{2} \Delta x^{3}\left(\mu+\mu_{t}\right)}{\hat{\boldsymbol{e}}^{\mathbf{1}} \cdot \Delta \boldsymbol{R}} \\
& =\frac{\sqrt{g} h^{1} \Delta x^{2} \Delta x^{3}\left(\mu+\mu_{t}\right)}{\frac{\boldsymbol{e}^{\mathbf{1}} \cdot\left(\Delta x^{1} \boldsymbol{e}_{\mathbf{1}}+\Delta x^{2} \boldsymbol{e}_{\mathbf{2}}+\Delta x^{3} \boldsymbol{e}_{\mathbf{3}}\right)}{h^{1}}} \\
& =\frac{\sqrt{g} \Delta x^{2} \Delta x^{3}\left(\mu+\mu_{t}\right) g^{11}}{\Delta x^{1}}
\end{aligned}
$$

Substituting for the primary fluxes from Eqs. (38) - (40) in Eq. (42) and using the convection, diffusion conductance of Eq. (43), the following equation is obtained.

$$
\begin{aligned}
\frac{\partial\left(\rho v_{1 P}\right)}{\partial t} \Delta \forall & +\left[\left(F v_{1}\right)_{e}-D_{e}\left(v_{1 E}-v_{1 P}\right)\right]-\left[\left(F v_{1}\right)_{w}-D_{w}\left(v_{1 P}-v_{1 W}\right)\right] \\
& +\left[\left(F v_{1}\right)_{n}-D_{n}\left(v_{1 N}-v_{1 P}\right)\right]-\left[\left(F v_{1}\right)_{s}-D_{s}\left(v_{1 P}-v_{1 S}\right)\right] \\
& +\left[\left(F v_{1}\right)_{t}-D_{t}\left(v_{1 T}-v_{1 P}\right)\right]-\left[\left(F v_{1}\right)_{b}-D_{b}\left(v_{1 P}-v_{1 B}\right)\right]=-A_{P}\left[p_{e}-p_{w}\right]+b_{v_{1 P}}
\end{aligned}
$$

$A_{P}$ denotes the area $\sqrt{g} h^{1} \Delta x^{2} \Delta x^{3}$. Defining the neighboring influence coefficients as

$$
\begin{aligned}
\bar{a}_{E} & =D_{e} A\left(\left|P e_{e}\right|\right)+\left[\left[-F_{e}, 0\right]\right] \\
\bar{a}_{W} & =D_{w} A\left(\left|P e_{w}\right|\right)+\left[\left[F_{w}, 0\right]\right]
\end{aligned}
$$

Similar terms can be defined for the other four neighbors. Pe is the cell Peclet number and is the equal to $F / D$. The function $A(|P e|)$ depends on the choice of the face flux scheme. In the present research, 
power law profile [13] is used. [[A,B]] denotes the maximum of two quantities $A, B$. With these influence coefficients, Eq. (46) can be rearranged as

$$
\frac{\partial\left(\rho v_{1 P}\right)}{\partial t} \Delta \forall+\bar{a}_{P} v_{1 P}=\Sigma \bar{a}_{n b} v_{1 n b}-A_{P}\left[p_{e}-p_{w}\right]+b_{v_{1 P}}
$$

with $\bar{a}_{P}$ being $\Sigma \bar{a}_{n b}$. Discretizing Eq. (49) in time yields,

$$
\begin{aligned}
\frac{\rho \Delta \forall}{\Delta t}\left(v_{1 P}-v_{1 P}^{0}\right)+\alpha \bar{a}_{P} v_{1 P} & =\alpha \Sigma \bar{a}_{n b} v_{1 n b}-A_{P}\left[p_{e}-p_{w}\right]+\alpha b_{v_{1 P}} \\
& +(1-\alpha)\left[\Sigma \bar{a}_{n b}^{0} v_{1 n b}^{0}+b_{v_{1 P}}^{0}-\bar{a}_{P}^{0} v_{1 P}^{0}\right]
\end{aligned}
$$

' $\alpha$ ' depends on the time integration scheme and takes the value of $0.5,1$ for Crank-Nicolson and fully implicit discretization respectively. The variables with the superscript ' 0 ' denote their values from the previous time-step. Pressure is treated fully implicitly. Equation (50) can be rearranged in the form

$$
a_{P} v_{1 P}=\Sigma a_{n b} v_{1 n b}+b_{P}-A_{P}\left[p_{e}-p_{w}\right]
$$

The various terms in the final discretized Eq. (51) are

$$
\begin{aligned}
a_{P} & =\alpha \bar{a}_{P}+\frac{\rho \Delta \forall}{\Delta t} \\
a_{n b} & =\alpha \bar{a}_{n b} \\
b_{P} & =\alpha b_{v_{1 P}}+(1-\alpha)\left[\Sigma \bar{a}_{n b}^{0} v_{1 n b}^{0}+b_{v_{1 P}}^{0}-\bar{a}_{P}^{0} v_{1 P}^{0}\right]+\frac{\rho \Delta \forall}{\Delta t} v_{1 P}^{0}
\end{aligned}
$$

The other momentum and turbulence transport equations can be discretized following a similar procedure. The turbulence quantities $\mathcal{K}$ and $\epsilon$ are non-negative. The secondary diffusive fluxes of $\mathcal{K}, \epsilon$ in Eqs. (33), (34) contain non-orthogonal metrics and gradients of turbulent quantity. These terms may become locally negative in certain regions of the domain during an iterative process. If the magnitude of the negative source contribution dominates the positive part, $\mathcal{K}$ and $\epsilon$ will acquire negative values. Proper source term linearization is necessary to ensure physically realistic solution [13]. If the source term $S_{P}$, for the variable $\phi_{P}$, consists of positive and negative contribution i.e. $S_{P}=S_{P}^{+}-S_{P}^{-}$, then it may be rearranged as

$$
S_{P}=S_{P}^{+}-\left(S_{P}^{-} \times \frac{\phi_{P}}{\phi_{P}}\right)=S_{P}^{+}-\left(S_{P}^{-} \times \frac{\phi_{P}}{\phi_{P}^{*}}\right)
$$

where $\phi_{P}^{*}$ is the previous iteration value of $\phi_{P}$. The negative source term is then lumped onto the central coefficient $a_{P}=a_{P}+S_{P}^{-} / \phi_{P}^{*}$

\subsection{Discretization of continuity equation}

The mass conservation Eq. (26) can be expanded and rearranged by moving the non-orthogonal terms to the RHS.

$$
\begin{aligned}
\frac{1}{\sqrt{g}} \frac{\partial}{\partial x^{1}}( & \left.\frac{\rho \sqrt{g} g^{11} v_{1}}{h^{1}}\right)+\frac{1}{\sqrt{g}} \frac{\partial}{\partial x^{2}}\left(\frac{\rho \sqrt{g} g^{22} v_{2}}{h^{2}}\right)+\frac{1}{\sqrt{g}} \frac{\partial}{\partial x^{3}}\left(\frac{\rho \sqrt{g} g^{33} v_{3}}{h^{3}}\right) \\
= & -\frac{1}{\sqrt{g}} \frac{\partial}{\partial x^{1}}\left[\rho \sqrt{g}\left(\frac{g^{12} v_{2}}{h^{2}}+\frac{g^{13} v_{3}}{h^{3}}\right)\right]-\frac{1}{\sqrt{g}} \frac{\partial}{\partial x^{2}}\left[\rho \sqrt{g}\left(\frac{g^{21} v_{1}}{h^{1}}+\frac{g^{23} v_{3}}{h^{3}}\right)\right] \\
& -\frac{1}{\sqrt{g}} \frac{\partial}{\partial x^{3}}\left[\rho \sqrt{g}\left(\frac{g^{31} v_{1}}{h^{1}}+\frac{g^{32} v_{2}}{h^{2}}\right)\right]
\end{aligned}
$$

The leading time derivative term is dropped on account of incompressibility. Denoting the non-orthogonal terms on the RHS by $b_{N O}$, and noting that $g^{11}=h^{1} h^{1}, g^{22}=h^{2} h^{2}, g^{33}=h^{3} h^{3}$,

$$
\frac{1}{\sqrt{g}} \frac{\partial}{\partial x^{1}}\left(\rho \sqrt{g} h^{1} v_{1}\right)+\frac{1}{\sqrt{g}} \frac{\partial}{\partial x^{2}}\left(\rho \sqrt{g} h^{2} v_{2}\right)+\frac{1}{\sqrt{g}} \frac{\partial}{\partial x^{3}}\left(\rho \sqrt{g} h^{3} v_{3}\right)=b_{N O}
$$


Integrating over a finite control volume $\Delta \forall=\sqrt{g} \Delta x^{1} \Delta x^{2} \Delta x^{3}$ and discretizing,

$$
\left(\rho A_{e} v_{1 e}\right)-\left(\rho A_{w} v_{1 w}\right)+\left(\rho A_{n} v_{2 n}\right)-\left(\rho A_{s} v_{2 s}\right)+\left(\rho A_{t} v_{3 t}\right)-\left(\rho A_{b} v_{3 b}\right)=b_{N O} \Delta \forall
$$

The area terms on the east, north and top faces are $A_{e}=\left(\sqrt{g} h^{1} \Delta x^{2} \Delta x^{3}\right)_{e}, A_{n}=\left(\sqrt{g} h^{2} \Delta x^{1} \Delta x^{3}\right)_{n}$, $A_{t}=\left(\sqrt{g} h^{3} \Delta x^{1} \Delta x^{2}\right)_{t}$. Similar area terms $A_{w}, A_{s}, A_{b}$ can be defined for the west, south and bottom faces.

\subsection{Pressure equation}

The pressure-velocity coupling is handled through the SIMPLER algorithm of Patankar [13]. The discrete equations of mass and momentum are manipulated to obtain an exact pressure equation and an approximate pressure-correction equation. The discrete equation for $v_{1}$ was obtained at a cell center point $P$ in Eq. (51). Instead, if the discrete equation for the $v_{1}$ is obtained at the cell face $e$, it would have the following form.

$$
a_{e} v_{1 e}=\Sigma a_{n b} v_{1 n b}+b_{e}-A_{e}\left[p_{E}-p_{P}\right]
$$

Dividing the above equation by the central coefficient $a_{e}$,

$$
v_{1 e}=\hat{v}_{1 e}+d_{e}\left[p_{P}-p_{E}\right]
$$

The quantity $\hat{v}_{1 e}=\left(\sum a_{n b} v_{1 n b}+b_{e}\right) / a_{e}$ is called pseudo-velocity. The term $d_{e}=A_{e} / a_{e}$, is the ratio of area to the central coefficient and is always a positive quantity. Similar equations can be obtained for the other two momentum equations at $n, t$ faces.

$$
\begin{aligned}
v_{2 n} & =\hat{v}_{2 n}+d_{n}\left[p_{P}-p_{N}\right] \\
v_{3 t} & =\hat{v}_{3 t}+d_{t}\left[p_{P}-p_{T}\right]
\end{aligned}
$$

Substituting Eqs. (60) - (62) (and analogous terms for $v_{1 w}, v_{2 s}, v_{3 b}$ ) into Eq. (58) and rearranging, the following exact equation for pressure is obtained.

$$
a_{P} p_{P}=\Sigma a_{n b} p_{n b}+\hat{b}_{P}+b_{N O} \Delta \forall
$$

where

$$
\begin{aligned}
a_{n b} & =\rho A_{n b} d_{n b} \\
a_{P} & =\Sigma a_{n b} \\
\hat{b}_{P} & =\left(\rho A_{w} \hat{v}_{1 w}\right)-\left(\rho A_{e} \hat{v}_{1 e}\right)+\left(\rho A_{s} \hat{v}_{2 s}\right)-\left(\rho A_{n} \hat{v}_{2 n}\right)+\left(\rho A_{b} \hat{v}_{3 b}\right)-\left(\rho A_{t} \hat{v}_{3 t}\right)
\end{aligned}
$$

The neighbors $n b$ include $E, W, N, S, T, B$ grid points. The non-orthogonal terms of the continuity equation constitute additional source term $b_{N O} \Delta \forall$ in the pressure equation. These terms are lagged by an iteration and treated explicitly [9]. The procedure for obtaining the pressure-correction equation closely follows the pressure equation [13]. It can be seen that the stencil for the discrete pressure equation involves only the central and neighbor points, resulting in the simplest possible stencil -5 points in $2 \mathrm{D}$ and 7 points in $3 \mathrm{D}$. The neighbor coefficients $a_{n b}$ are always positive and consequently the central coefficient $a_{P}$ is never lower than $\Sigma\left|a_{n b}\right|$. Thus, the discrete pressure equation is compact and diagonally dominant. In contrast, if non-directional pressure gradient terms are present (as in Cartesian or contravariant formulation), the discrete pressure equation will have larger stencil. The coefficients may be positive or negative depending on the local grid non-orthogonality and diagonal dominance is no longer guaranteed (see Eq. (D.12)).

The discretized equations form a banded matrix and are solved using a line-by-line Tri-Diagonal-Matrix Algorithm (TDMA) and a Gauss-Seidel iterative scheme. On each ' $i$ ' plane, the equations are solved using TDMA along the positive and negative ' $j$ ', ' $k$ ' direction. The process is repeated in cyclic order for ' $j$ ', ' $k$ ' planes to complete one sweep. Multiple sweeps are necessary to solve the simultaneous algebraic equations. In the current procedure, 5 sweeps are used for solving the pressure, pressure correction, turbulence equations and 3 sweeps are used for solving the momentum equations. During the computational process, the RANS equation are solved first. Multiple iterations of pressure, momentum and pressure-correction are performed successively until convergence is achieved in each time-step. The converged flow field is then used to solve the turbulence equations. The wall functions are implemented in a manner similar to Sondak and Pletcher [16]. 


\section{Results}

\subsection{D laminar flow in a lid driven skewed cavity}

The 2D laminar flow in a lid driven skewed cavity has been widely analyzed [4, 17-26]. Literature related to this problem can be classified into two broad categories - those that seek to provide a benchmark solution to the skewed cavity problem, and those that test different formulations of the Navier-Stokes equation. The former usually adopts the vorticity-streamfunction approach in which the pressure field is not solved in the intermediate steps. Failure of convergence in an incompressible flow is often attributed to the resolution of the pressure field. As the vorticity-streamfunction form does not involve pressure, the algorithm is comparatively stable and an accurate benchmark solution can be obtained for different Reynolds numbers even at extreme skew angles. Erturk and Dursun [26] used this approach and reported the results on a $512 \times 512$ grid for a variety of skew angles, ranging from the perfectly orthogonal square cavity to an extremely skewed non-orthogonal cavity.

Researchers who test different formulations of the Navier-Stokes equation use the primitive pressurevelocity approach. As reviewed in the introduction section, the diagonal dominance of the pressure equation and consequently the convergence depends on the individual formulation. Peric [4] used the contravariant velocity as the primary unknown and a simplified stencil for the pressure equation by ignoring the cross derivative pressure terms. He concluded that the simplified stencil (5 points in 2D and 7 points in 3D) becomes inefficient when the grid skewness approaches $45^{\circ}$ and fails to converge for angles below $30^{\circ}$. A complex stencil for the pressure equation (9 points in 2D and 19 points in 3D) was necessary for convergence at extreme skew angles. Most of the literature that use the primitive pressure-velocity equation with the Cartesian and contravariant velocity as the unknown, report the results only at moderately skewed angles without investigating the convergence at extreme angles [17-25]. Convergence characteristics of skewed cavity at extreme angles with covariant velocity as the main variable are presented here. The problem geometry is described in Fig. 3. The length of the cavity along each edge is $L$. The angle between the edges of the cavity is $\gamma$. By changing the angle $\gamma$, the grid non-orthogonality can be varied.

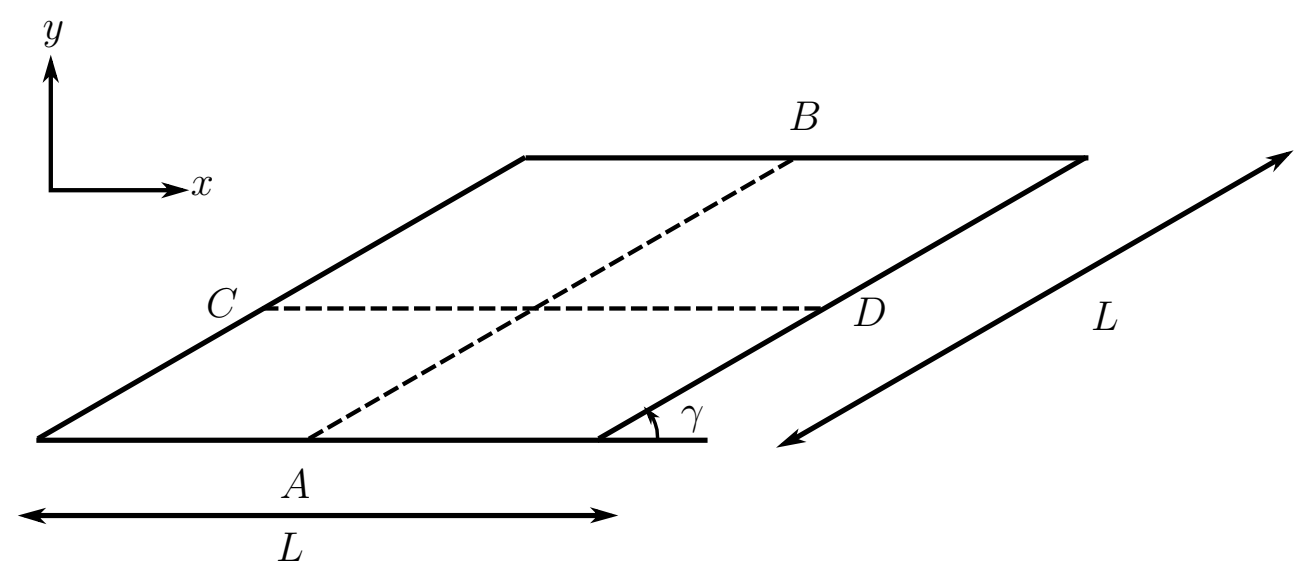

Fig. 3. Lid driven skewed cavity.

All the boundaries except the top, is a viscous no-slip wall. The fluid velocity at the top boundary is $U_{o}$ in the Cartesian $x$ direction. Laminar flow at $R e=\rho U_{o} L / \mu=100$ and 1000 for different skew angles were studied. The Cartesian $u$ velocity along the line $A-B$ and $v$ velocity along the line $C-D$ were used for comparison. These lines are located at $50 \%$ distance along the corresponding edges. During the analysis, it was found that the $v$ velocity profile along the line $C-D$ was more sensitive to the grid size, especially at extreme skew angles and higher Reynolds numbers. Therefore, a grid independence study was performed at $\gamma=150^{\circ}$ and $R e=1000$ with varying uniform grids of size $32 \times 32,64 \times 64,128 \times 128,256 \times 256$ and $275 \times 275$. Figures $4 \mathrm{a}, 4 \mathrm{~b}$ show the comparison of the velocity profiles for different grid sizes. It can be seen 
that the velocity profiles corresponding to the size $256 \times 256$ and $275 \times 275$ are identical and matches well with the $512 \times 512$ simulation of Erturk and Dursun [26]. The $256 \times 256$ grid is thus considered to provide a grid independent solution from the present simulation and all the results presented henceforth correspond to this grid size.

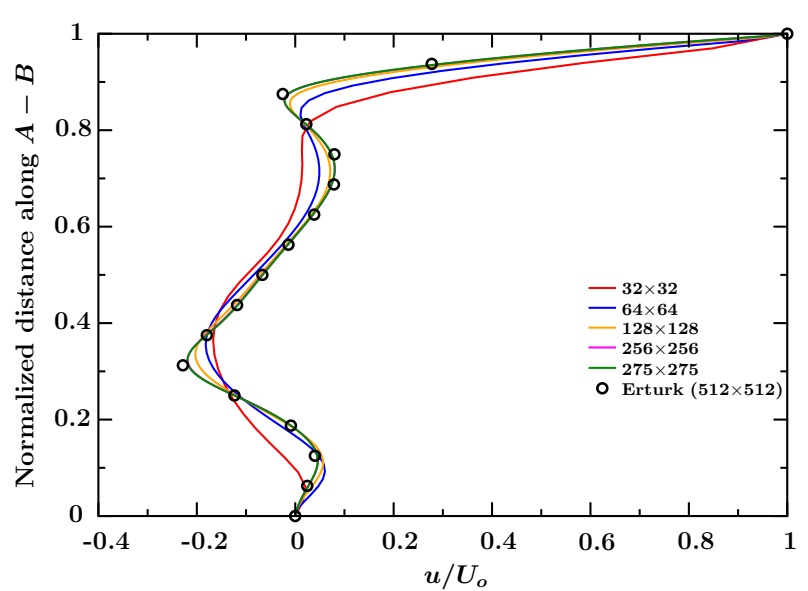

(a) $u$ velocity

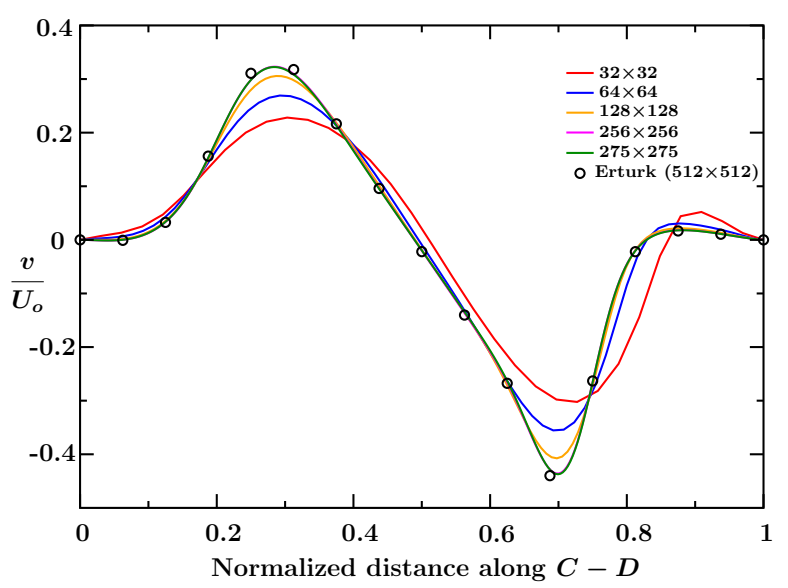

(b) $v$ velocity

Fig. 4. Grid independence study at $\operatorname{Re}=1000, \gamma=150^{\circ}$.

Several cases were run at Reynolds numbers 100 and 1000 for various skew angles ranging from $\gamma=15^{\circ}$ to $\gamma=165^{\circ}$ in increments of $\Delta \gamma=15^{\circ}$. These encompass the perfectly orthogonal square cavity of $\gamma=90^{\circ}$ and the extremely skewed cavities of $\gamma=15^{\circ}$ and $\gamma=165^{\circ}$. In each case, the present simulation compared well with the tabulated values of Erturk and Dursun [26]. For want of space, only the extremely skewed cases of $\gamma=15^{\circ}$ and $\gamma=165^{\circ}$ are presented here for the two Reynolds numbers. Figures 5a, 5b and Figs. 6a, 6b show the comparison of the velocity profiles at $R e=100$ for $\gamma=15^{\circ}$ and $\gamma=165^{\circ}$ respectively. The corresponding comparison for $R e=1000$ is plotted in Figs. 7a, 7b and Figs. 8a, 8b. These favorable comparisons vis-à-vis the conclusions of Peric [4] establish that using covariant velocity as the primary unknown leads to a diagonally dominant pressure equation with a simplified stencil that favors convergence even in extremely non-orthogonal grids.

In order to estimate the accuracy of present simulations, four error parameters are computed and monitored for the various grids. These error parameters are the mass flow rate $\dot{m}$ and $L_{2}$ norm for $u, v$ velocity along the lines $A-B$ and $C-D$. Since this is an incompressible flow and there is no net mass flow into or out of the domain, the mass flow rate along the two lines should identically be equal to zero [26]. The absolute value of the mass flow rate along $A-B$ and $C-D$ are given by,

$$
\begin{aligned}
& \dot{m}_{A B}=\left|\int_{A}^{B} \rho \boldsymbol{V} \cdot \boldsymbol{d} \boldsymbol{A}\right|=\left|\int_{A}^{B} \rho(u d y-v d x)\right| \\
& \dot{m}_{C D}=\left|\int_{C}^{D} \rho \boldsymbol{V} \cdot \boldsymbol{d} \boldsymbol{A}\right|=\left|\int_{C}^{D} \rho v d x\right|
\end{aligned}
$$

The $L_{2}$ error of $u, v$ velocities along $A-B$ and $C-D$ are calculated as,

$$
\begin{aligned}
L_{2-u-A B} & =\sqrt{\frac{\sum_{i=1}^{N}\left(u_{\text {exact }}-u\right)^{2}}{N}} \\
L_{2-v-C D} & =\sqrt{\frac{\sum_{i=1}^{N}\left(v_{\text {exact }}-v\right)^{2}}{N}}
\end{aligned}
$$




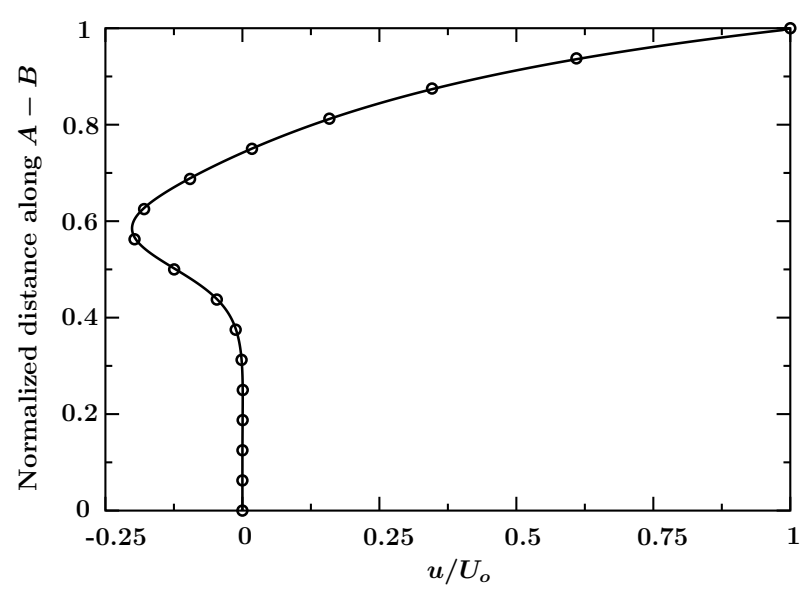

(a) $u$ velocity

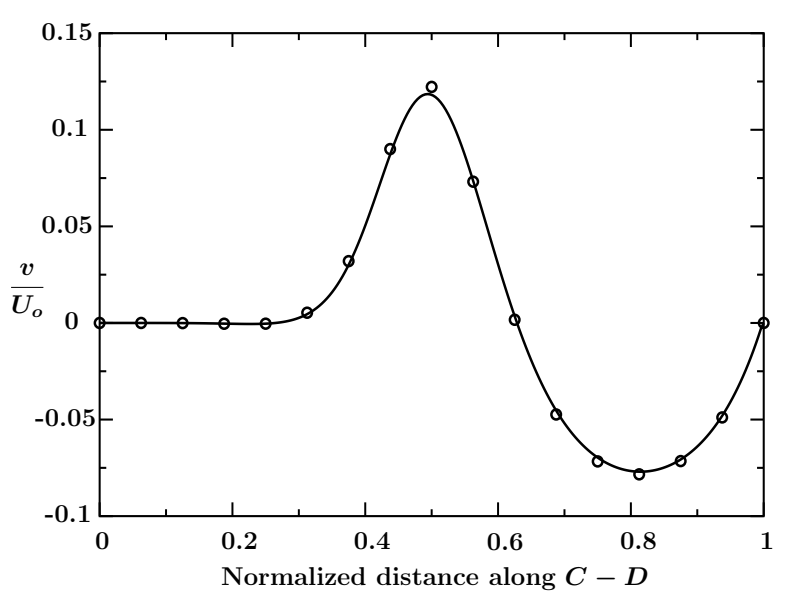

(b) $v$ velocity

Fig. 5. Variation of velocity along the centerline. $R e=100, \gamma=15^{\circ}$. Present simulation ( - ), Erturk and Dursun [26] simulation $(\circ)$

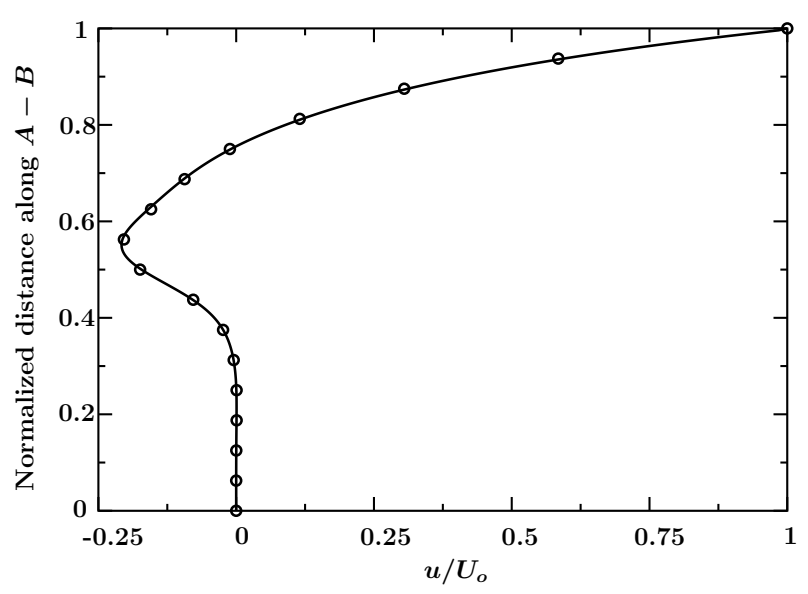

(a) $u$ velocity

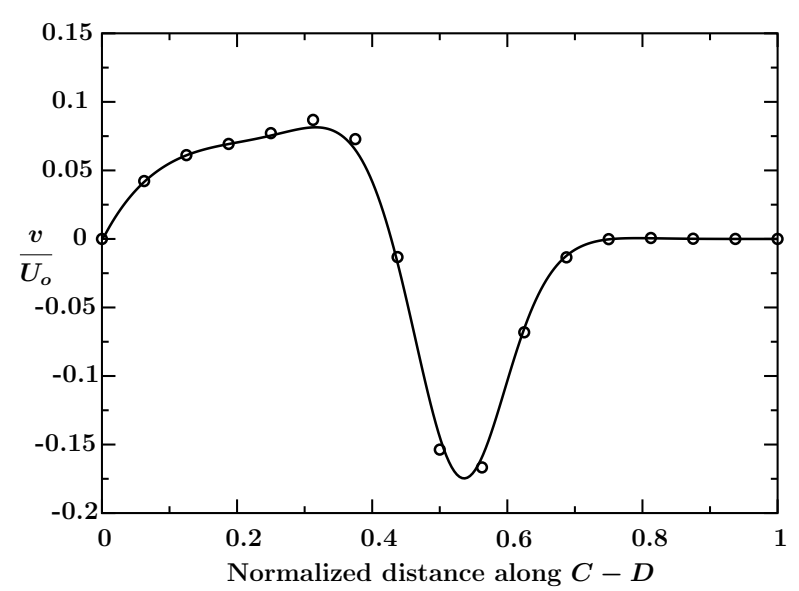

(b) $v$ velocity

Fig. 6. Variation of velocity along the centerline. $R e=100, \gamma=165^{\circ}$. Present simulation ( - ),Erturk and Dursun [26] simulation $(\circ)$

where $u_{\text {exact }}, v_{\text {exact }}$ are from [26] and $u, v$ are from the present simulation. The benchmark results $u_{\text {exact }}$, $v_{\text {exact }}$ are available at seventeen equidistant points. The present $u, v$ values from the different grids are interpolated to the same locations as $u_{\text {exact }}$, $v_{\text {exact }}$ using a second order B-spline interpolation technique and the $L_{2}$ errors are calculated using Eqs. (69), (70). To calculate the mass flow rate $\dot{m}$, the velocities $u, v$ are interpolated to 1000 points and the integration in Eq. (67), (68) is carried out numerically using trapezoidal rule. The mass flow rate $\dot{m}_{A B}, \dot{m}_{C D}$ give an estimate of the accuracy of the present simulation against expected values, whereas $L_{2-u-A B}, L_{2-v-C D}$ indicate how closely the present simulation matches with that of [26]. The error values for the different grids are tabulated for $\gamma=15^{\circ}, \gamma=165^{\circ}$ at $R e=100, R e=1000$ in Tables 1 - 4. It can be seen that as the grid is refined, the errors are progressively reduced. The absolute value of the mass flow rates for the $256 \times 256$ grid are in the range $10^{-5}-10^{-6}$ and the $L_{2}$ errors are in the range $10^{-3}-10^{-4}$. 




(a) $u$ velocity

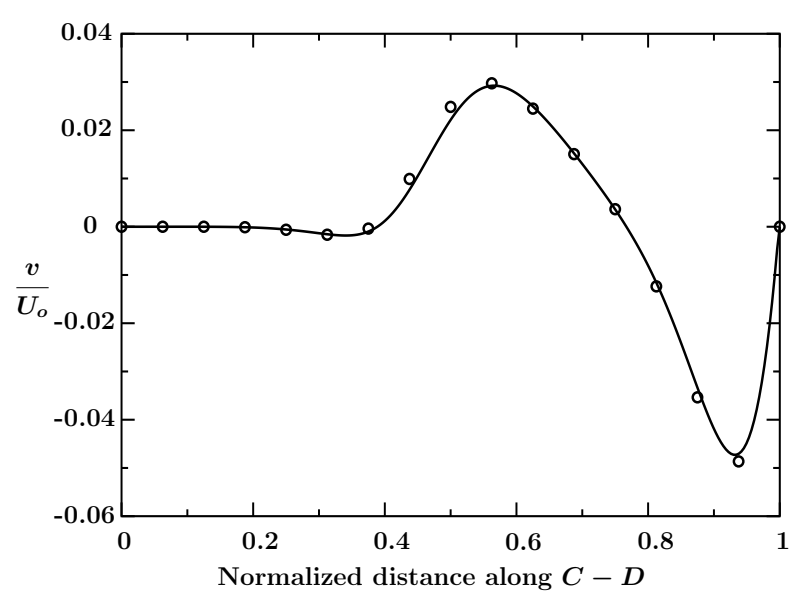

(b) $v$ velocity

Fig. 7. Variation of velocity along the centerline. $R e=1000, \gamma=15^{\circ}$. Present simulation ( — ), Erturk and Dursun [26] simulation $(\circ)$

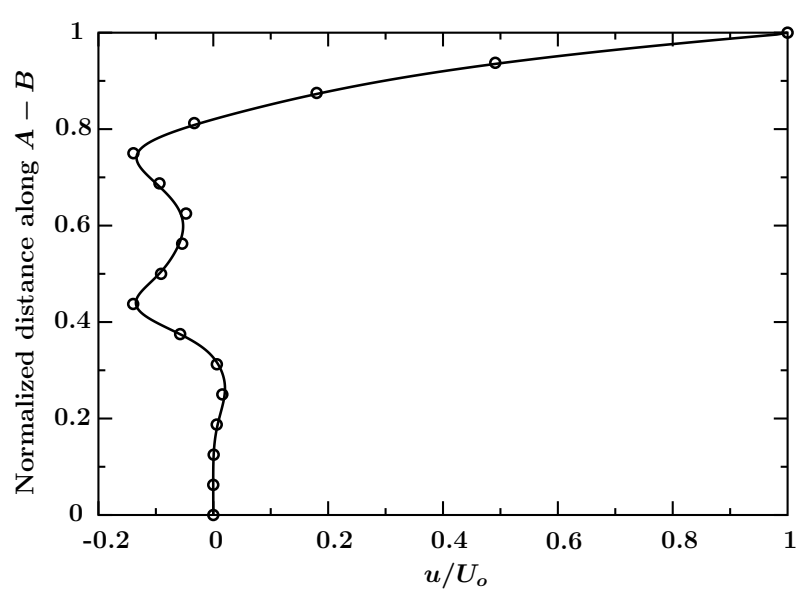

(a) $u$ velocity

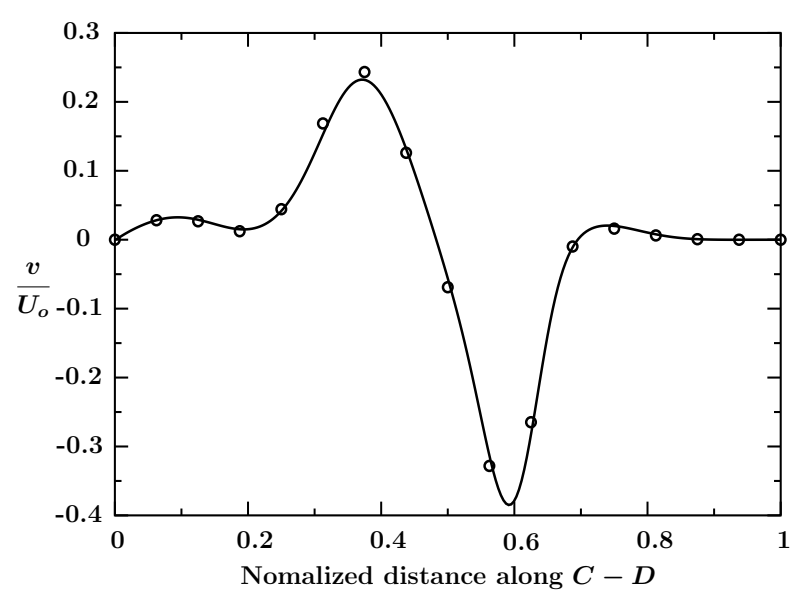

(b) $v$ velocity

Fig. 8. Variation of velocity along the centerline. $R e=1000, \gamma=165^{\circ}$. Present simulation ( - ),Erturk and Dursun [26] simulation $(\circ)$

Table 1. Errors in mass flow rate along $A-B, C-D$ for $R e=100$

\begin{tabular}{|c|c|c|c|c|}
\hline \multirow{2}{*}{ Grid size } & \multicolumn{2}{|c|}{$\gamma=15^{\circ}$} & \multicolumn{2}{c|}{$\gamma=165^{\circ}$} \\
\cline { 2 - 5 } & $\dot{m}_{A B}$ & $\dot{m}_{C D}$ & $\dot{m}_{A B}$ & $\dot{m}_{C D}$ \\
\hline $32 \times 32$ & $1.7039 \times 10^{-3}$ & $2.1254 \times 10^{-3}$ & $1.0010 \times 10^{-3}$ & $2.0490 \times 10^{-3}$ \\
\hline $64 \times 64$ & $4.6316 \times 10^{-4}$ & $5.1320 \times 10^{-4}$ & $2.9347 \times 10^{-4}$ & $4.5709 \times 10^{-4}$ \\
\hline $128 \times 128$ & $1.1981 \times 10^{-4}$ & $1.2391 \times 10^{-4}$ & $7.9498 \times 10^{-5}$ & $1.0977 \times 10^{-4}$ \\
\hline $256 \times 256$ & $3.0017 \times 10^{-5}$ & $3.0394 \times 10^{-5}$ & $2.0072 \times 10^{-5}$ & $2.7171 \times 10^{-5}$ \\
\hline
\end{tabular}


Table 2. $L_{2}$ errors in $u, v$ velocity along $A-B, C-D$ for $R e=100$

\begin{tabular}{|c|c|c|c|c|}
\hline \multirow{2}{*}{ Grid size } & \multicolumn{2}{|c|}{$\gamma=15^{\circ}$} & \multicolumn{2}{c|}{$\gamma=165^{\circ}$} \\
\cline { 2 - 5 } & $L_{2-u-A B}$ & $L_{2-v-C D}$ & $L_{2-u-A B}$ & $L_{2-v-C D}$ \\
\hline $32 \times 32$ & $4.8184 \times 10^{-2}$ & $2.1044 \times 10^{-2}$ & $5.2962 \times 10^{-2}$ & $1.9295 \times 10^{-2}$ \\
\hline $64 \times 64$ & $1.7970 \times 10^{-2}$ & $8.4943 \times 10^{-3}$ & $1.9977 \times 10^{-2}$ & $1.0343 \times 10^{-2}$ \\
\hline $128 \times 128$ & $7.0341 \times 10^{-3}$ & $2.8573 \times 10^{-3}$ & $7.9737 \times 10^{-3}$ & $4.4163 \times 10^{-3}$ \\
\hline $256 \times 256$ & $2.8614 \times 10^{-3}$ & $9.7046 \times 10^{-4}$ & $3.2981 \times 10^{-3}$ & $1.9139 \times 10^{-3}$ \\
\hline
\end{tabular}

Table 3. Errors in mass flow rate along $A-B, C-D$ for $R e=1000$

\begin{tabular}{|c|c|c|c|c|}
\hline \multirow{2}{*}{ Grid size } & \multicolumn{2}{|c|}{$\gamma=15^{\circ}$} & \multicolumn{2}{c|}{$\gamma=165^{\circ}$} \\
\cline { 2 - 5 } & $\dot{m}_{A B}$ & $\dot{m}_{C D}$ & $\dot{m}_{A B}$ & $\dot{m}_{C D}$ \\
\hline $32 \times 32$ & $5.7882 \times 10^{-3}$ & $3.3115 \times 10^{-3}$ & $4.4194 \times 10^{-3}$ & $1.4621 \times 10^{-3}$ \\
\hline $64 \times 64$ & $1.6449 \times 10^{-4}$ & $3.8579 \times 10^{-4}$ & $2.7122 \times 10^{-4}$ & $1.4939 \times 10^{-4}$ \\
\hline $128 \times 128$ & $5.6655 \times 10^{-5}$ & $1.3726 \times 10^{-4}$ & $7.3494 \times 10^{-5}$ & $2.8432 \times 10^{-5}$ \\
\hline $256 \times 256$ & $1.7600 \times 10^{-5}$ & $2.4434 \times 10^{-5}$ & $1.9171 \times 10^{-5}$ & $3.6926 \times 10^{-6}$ \\
\hline
\end{tabular}

Table 4. $L_{2}$ errors in $u, v$ velocity along $A-B, C-D$ for $R e=1000$

\begin{tabular}{|c|c|c|c|c|}
\hline \multirow{2}{*}{ Grid size } & \multicolumn{2}{|c|}{$\gamma=15^{\circ}$} & \multicolumn{2}{c|}{$\gamma=165^{\circ}$} \\
\cline { 2 - 5 } & $L_{2-u-A B}$ & $L_{2-v-C D}$ & $L_{2-u-A B}$ & $L_{2-v-C D}$ \\
\hline $32 \times 32$ & $9.8878 \times 10^{-2}$ & $1.4428 \times 10^{-2}$ & $7.8554 \times 10^{-2}$ & $9.5876 \times 10^{-2}$ \\
\hline $64 \times 64$ & $6.5336 \times 10^{-2}$ & $1.1043 \times 10^{-2}$ & $3.9661 \times 10^{-2}$ & $4.3025 \times 10^{-2}$ \\
\hline $128 \times 128$ & $1.4531 \times 10^{-2}$ & $3.9916 \times 10^{-3}$ & $1.6171 \times 10^{-2}$ & $1.8205 \times 10^{-2}$ \\
\hline $256 \times 256$ & $3.3469 \times 10^{-3}$ & $1.0749 \times 10^{-3}$ & $6.0129 \times 10^{-3}$ & $7.0858 \times 10^{-3}$ \\
\hline
\end{tabular}

An analysis on the number of iterations of the linear solver is carried out for the same test case as the earlier grid independence study i.e. $R e=1000, \gamma=150^{\circ}$. Three sets of simulations - Set 1 , Set 2, Set 3 are obtained by solving each linear equation once, five and ten times respectively. Upon convergence, all of the simulations gave the same final solution. The normalized residual of the pressure equation is plotted for the different iterations of the linear solver in Fig. 9. The residual is calculated as,

$$
R e s_{p}=\left|\sum a_{n b} p_{n b}+\hat{b}_{p}+b_{N O} \Delta \forall-a_{P} p_{P}\right|
$$

The residual is then normalized by dividing it with the residual value of the first iteration. It can be seen that as the number of iterations of the linear solver is increased, the convergence rate is enhanced. Finally, the mass residual history is plotted in Figs. 10a, 10b. Logarithm (to base 10) of the normalized value of the maximum absolute mass residual is plotted against the iteration number for the various obtuse angles of $\gamma$. It can be seen that the orthogonal square cavity converges fastest and the convergence rate slows down with increasing non-orthogonality. It must be noted here that all the cases of $\gamma$ except the extreme angles of $15^{\circ}$ and $165^{\circ}$ were run at a relaxation value of 0.4 for the momentum equations. The two extreme angles were simulated at a lower relaxation value of 0.2 for accurate convergence. Within the scope of the current research, analysis on the optimal value of relaxation factors was not undertaken. 


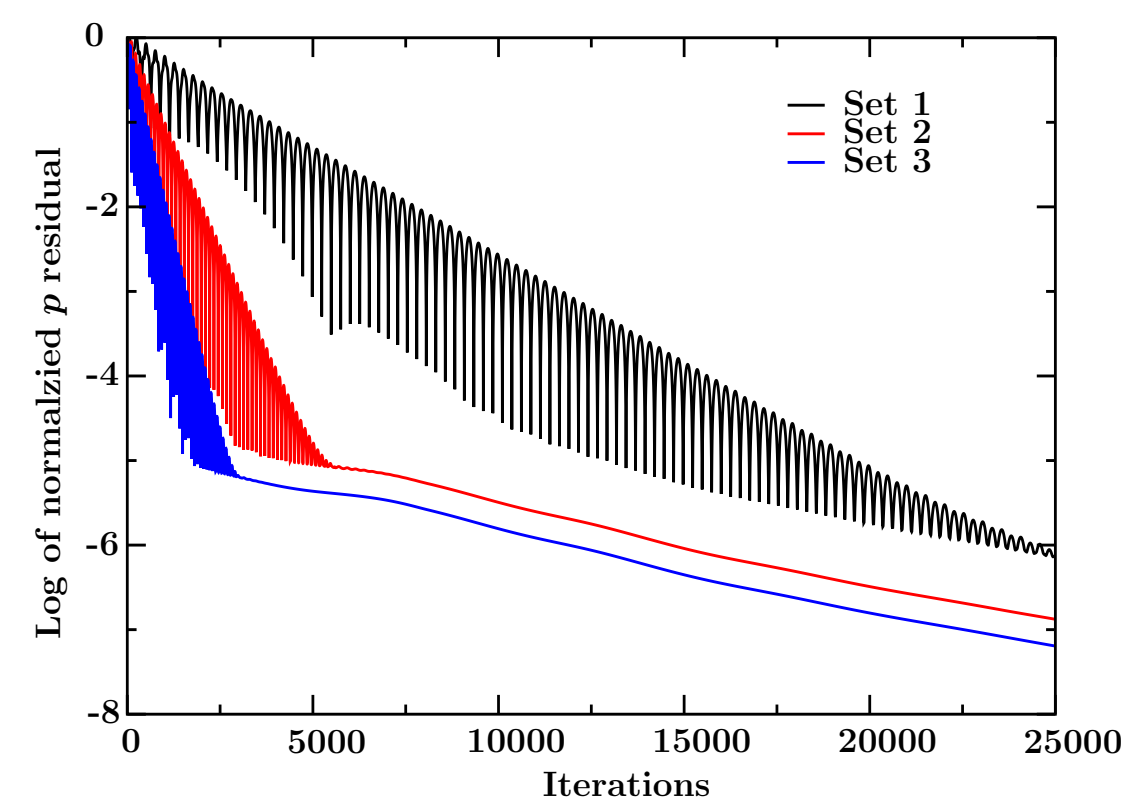

Fig. 9. Effect of number of iterations of the linear solver on the pressure equation residual.

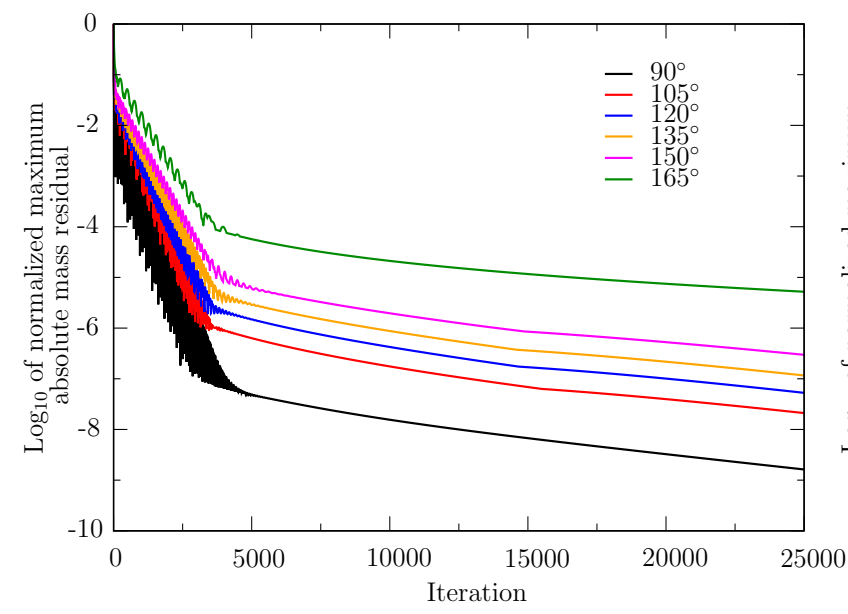

(a) Re 100

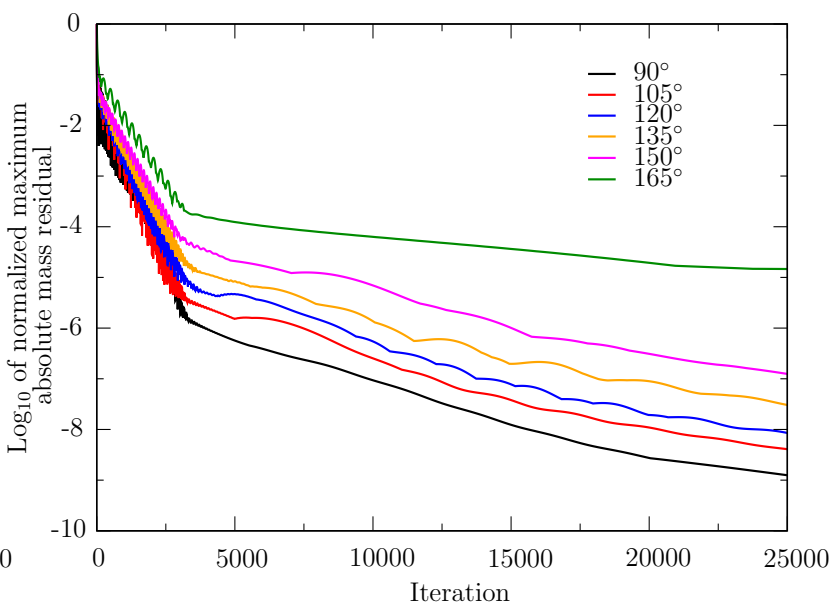

(b) Re 1000

Fig. 10. Comparison of residual history for different values of $\gamma$.

\subsection{D laminar flow in a $90^{\circ}$ L-bend}

3D laminar flow in a $90^{\circ}$ L-bend in simulated next. The geometry of the problem is described in Fig. 11. The duct consists of a square cross-section of size $W=1$ and $H=1$ unit. The inlet length $L_{i n}$ of the duct is 10 units. It is followed by a $90^{\circ}$ bend of inner and outer radius $r_{i}=1.8$ and $r_{o}=2.8$ units respectively. The outlet length $L_{\text {out }}$ is 5 units. The four walls of the duct are viscous no-slip walls. A fully developed laminar velocity profile obtained from a separate simulation of a long channel with same cross-section is specified at the inlet boundary. At the exit section, the boundary condition is imposed such that the stream-wise gradient of the velocity is zero and global mass conservation is satisfied. The Reynolds number based on the hydraulic diameter $R_{H}$ and the inlet bulk velocity $U_{B}$ is $R e=\rho U_{B} R_{H} / \mu=790$. A $160 \times 40 \times 40$ grid is used for the present simulation. The grids are uniform in the stream-wise direction and clustered near the walls. 


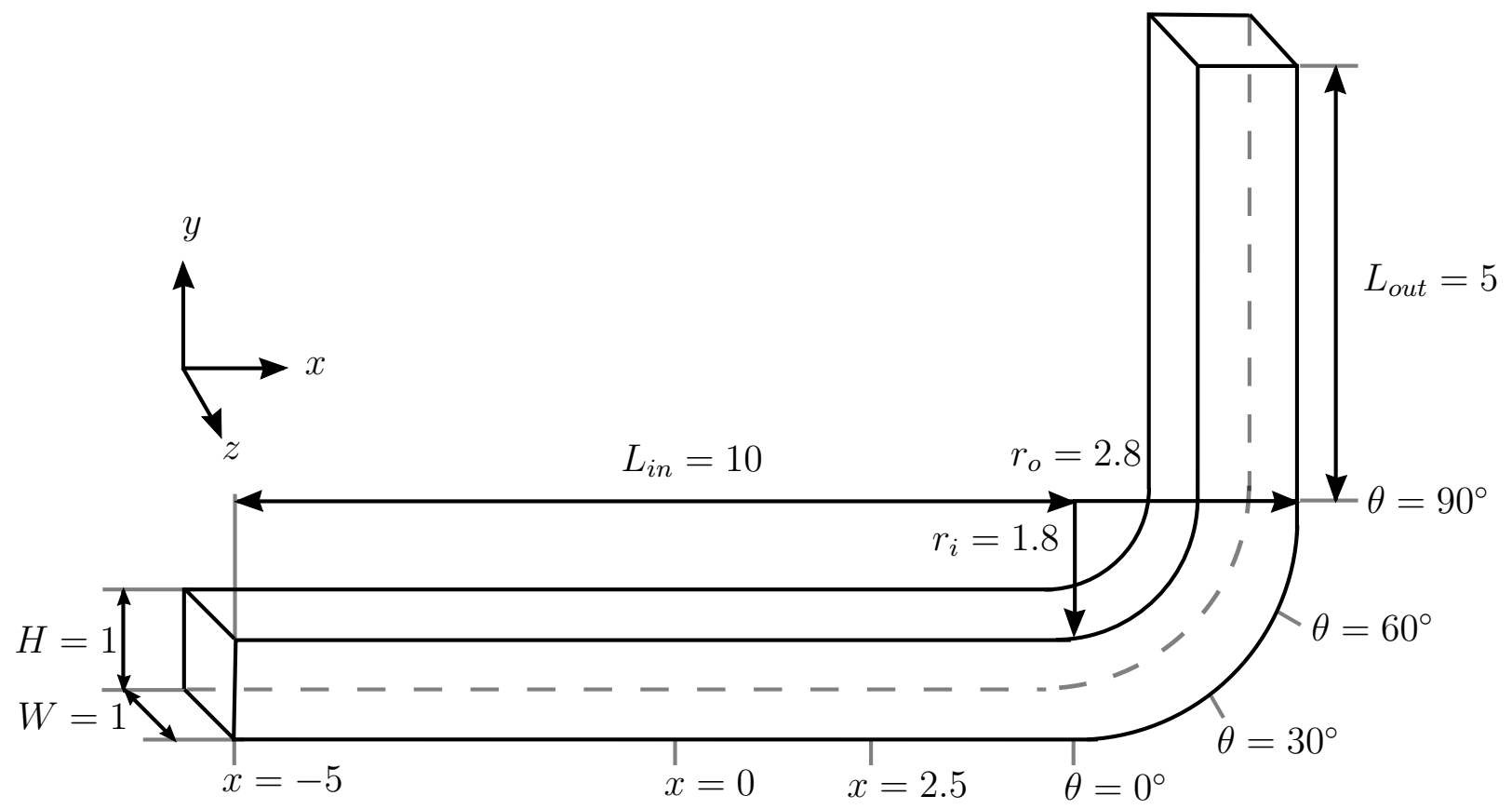

Fig. 11. $90^{\circ}$ L-bend with square cross-section.

Figures $12 \mathrm{a}, 12 \mathrm{~b}$ shows the comparison of the steady state stream-wise velocity profiles for six different stream-wise locations $x=0,2.5, \theta=0^{\circ}, 30^{\circ}, 60^{\circ}$, and $90^{\circ}$ at two span wise sections $z / W=0.25$ and 0.5 . In these figures, each velocity profile is offset from the previous station by a distance of 2.5 units along the vertical axis. The profiles are compared against the computational results (using contravariant flux as primary variable) of Rosenfeld et al. [27] and the experimental results of Humphrey et al. [28]. At the first three stations, there is no duct curvature and the velocity profile is symmetrical in the radial direction. There are no discernible differences between the present simulation and the results of [27, 28]. Within the L-bend, owing to the duct curvature, the fluid moves towards the outer radius, resulting in asymmetric velocity profile. The sections at $\theta=30^{\circ}, 60^{\circ}$, and $90^{\circ}$ are strongly affected by the curvature. At these locations, on comparison with the experimental results of Humphrey et al. [28], the present simulation matches more closely than the numerical results of Rosenfeld et al. [27]. 


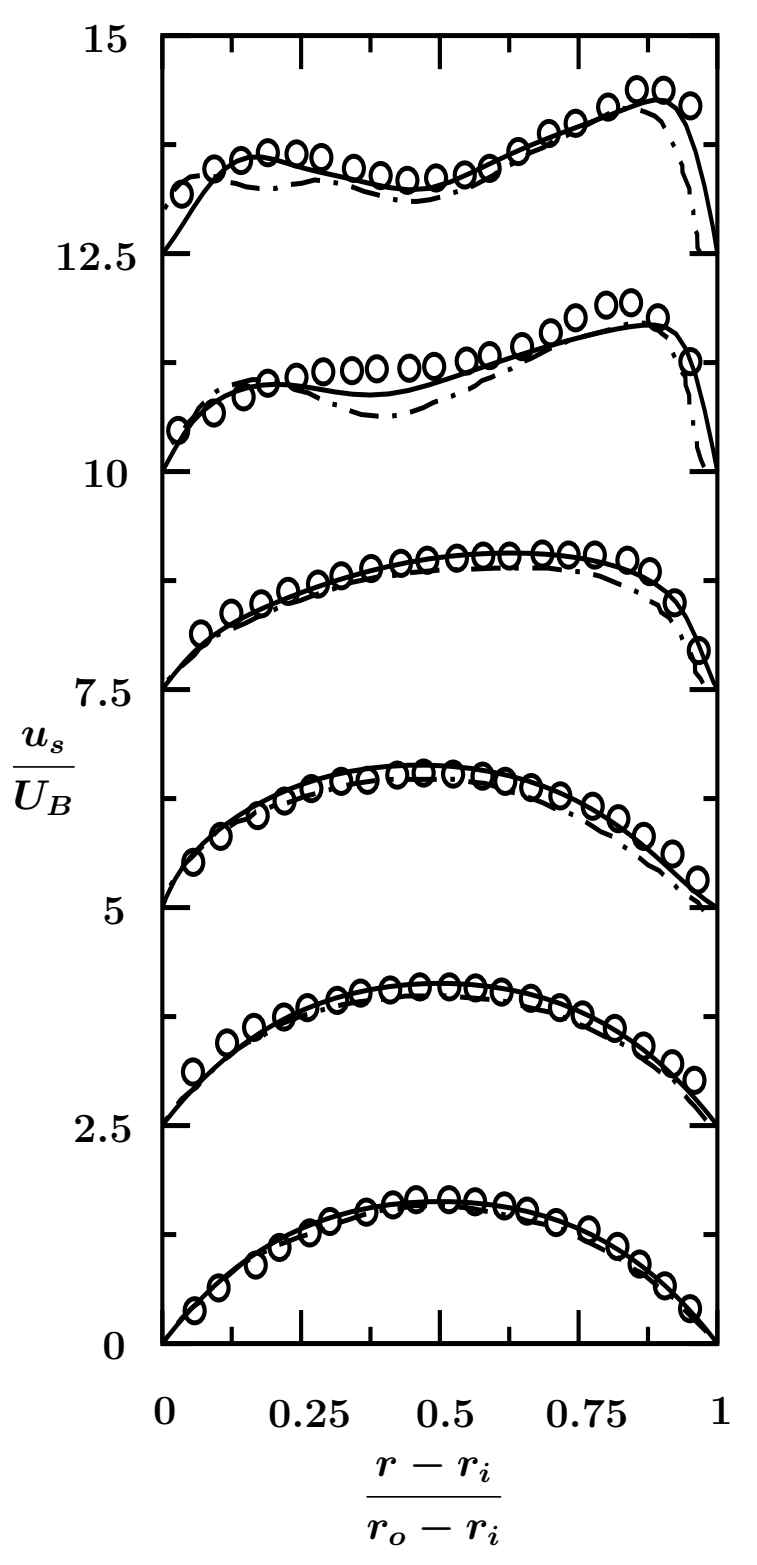

(a) $z / W=0.25$

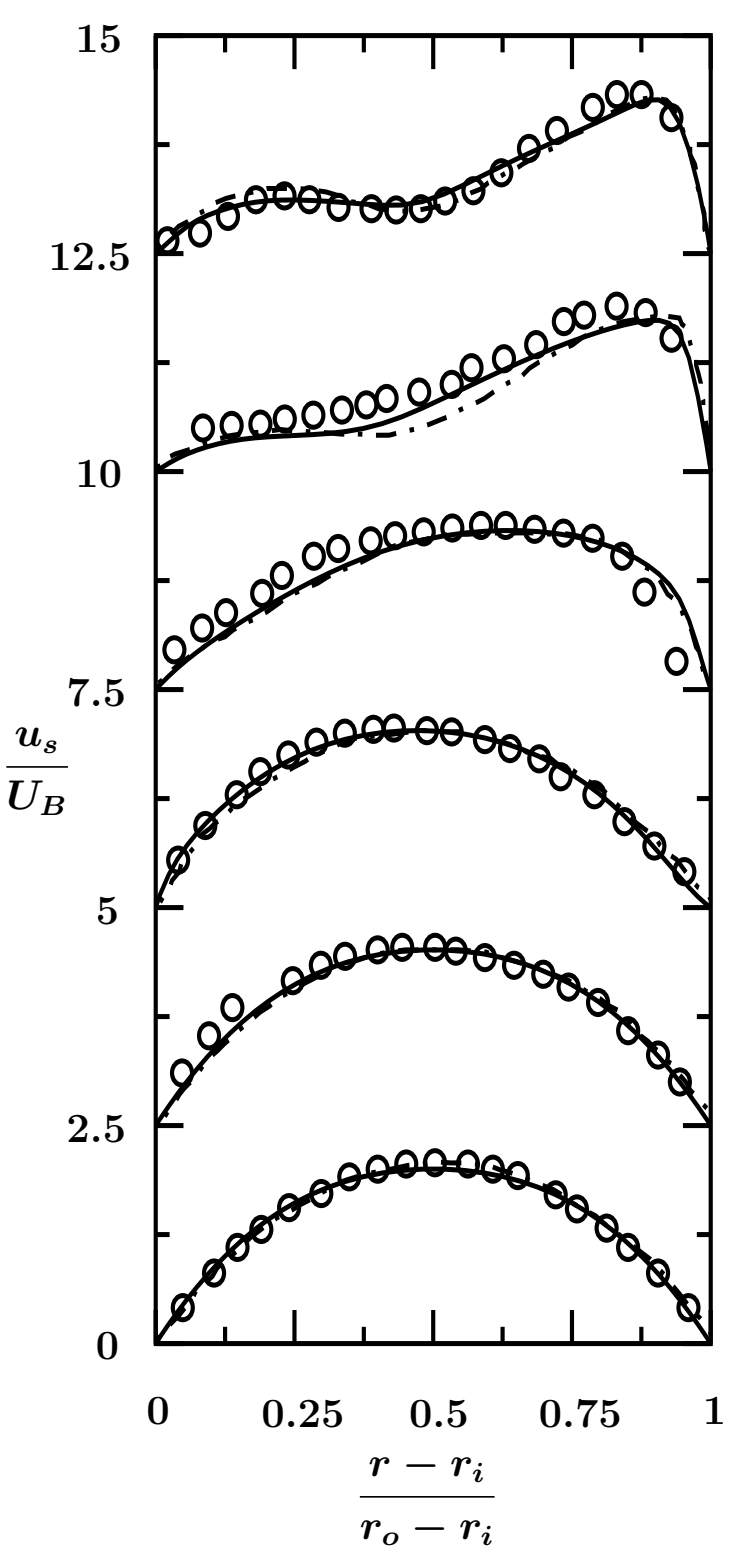

(b) $z / W=0.5$

Fig. 12. Stream-wise velocity profiles at six different stream-wise locations $x=0,2.5, \theta=0^{\circ}, 30^{\circ}, 60^{\circ}$, and $90^{\circ}$. Present computation $(-)$

Rosenfeld et al. [27] computation ( - - - ), Humphrey et al. [28] experiment ( ० )

A strong secondary flow is developed in the cross-sectional plane that is characterized by the motion of fluid towards and away from the sidewalls near the outer radius and inner radius respectively. This secondary flow pattern generates stream-wise vortices. The velocity vectors on the cross-sectional plane for the six stream-wise sections are shown in Figs. 13a - 13f. At $x=0$ and $x=2.5$ sections, the stream-wise flow dominates and the cross-sectional flow is negligible. At $\theta=0^{\circ}$ section, the flow starts to curl around and clear recognizable vortex patterns are seen at the $\theta=30^{\circ}$ and $\theta=60^{\circ}$ sections. At $\theta=90^{\circ}$ section, each half of the cross-sectional plane is characterized by three vortices - a dominant larger vortex near the inner radius, a second vortex near the outer radius, and a weaker smaller vortex near the symmetry plane. 


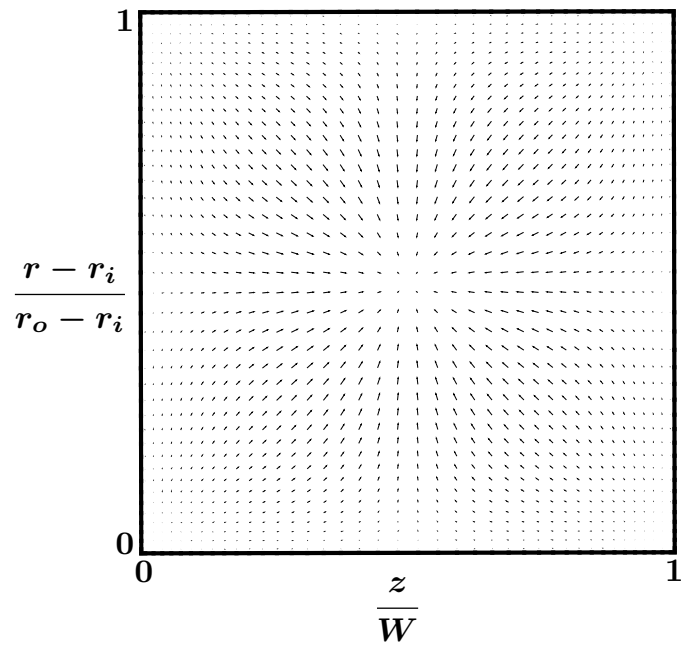

(a) $x=0$

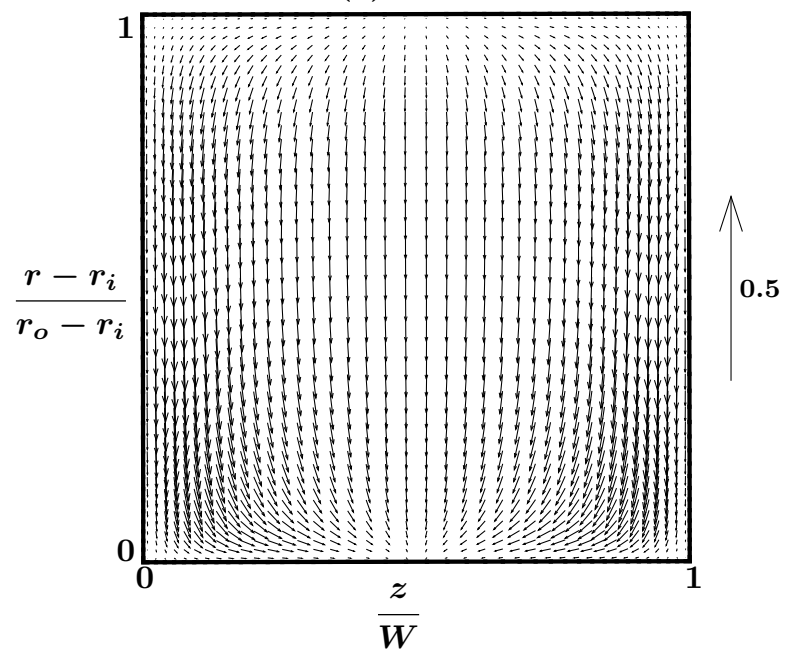

(c) $\theta=0^{\circ}$

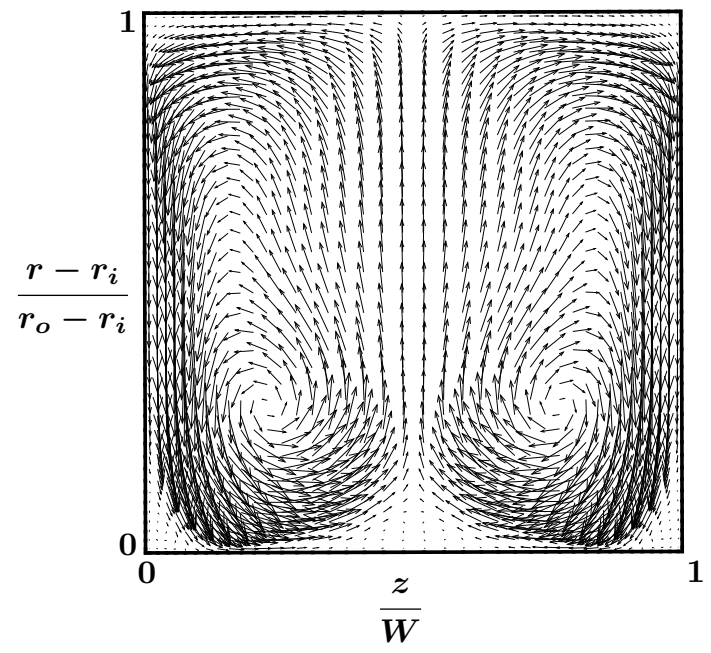

(e) $\theta=60^{\circ}$

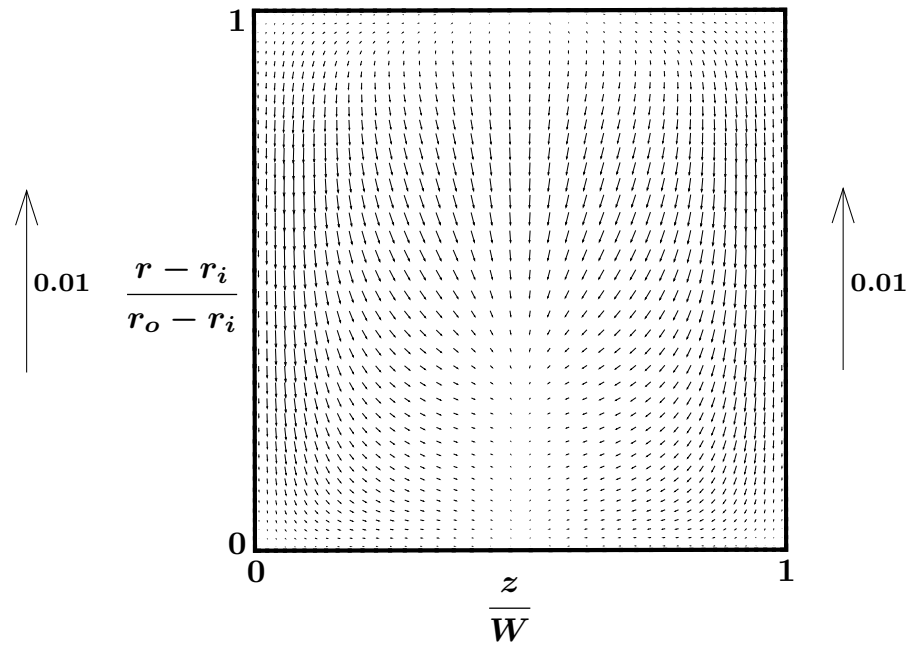

(b) $x=2.5$

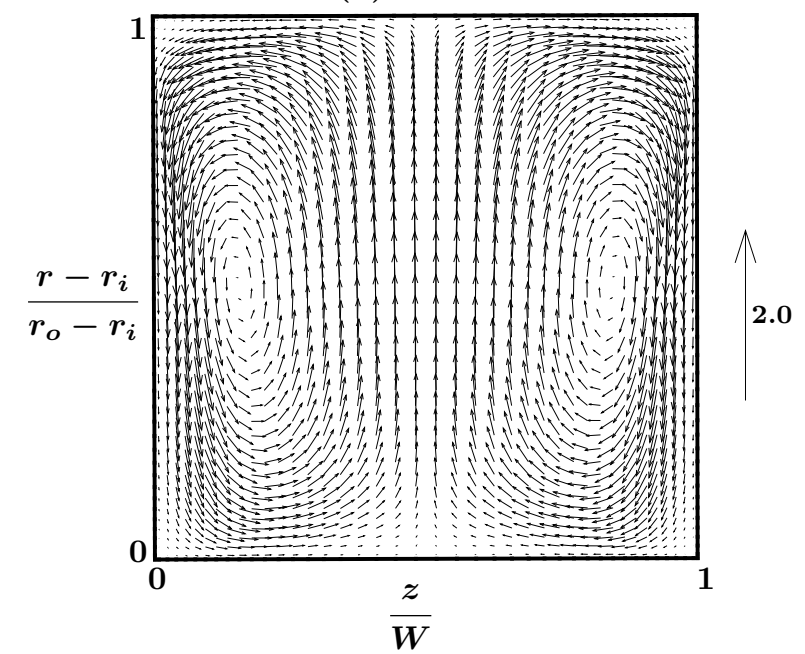

(d) $\theta=30^{\circ}$



(f) $\theta=90^{\circ}$

Fig. 13. Secondary flow velocity vectors on the cross-sectional plane at the six stream-wise locations. 


\subsection{Turbulent flow over $2 D$ model hill}

The ultimate objective of the present research is to develop a computational tool for studying the terrainwake interaction in a complex wind farm. In this context, turbulent flow over a hill is studied. The hill configuration corresponds to Almeida et al. [29] experiments. The profile of the hill is that of the plane of symmetry of the 3D hill used by Hunt and Snyder [30]. The profile is the inverse of a fourth order polynomial and was obtained from [31]. The convex and concave regions of the hill cause the flow to accelerate near the peak, followed by flow separation due to adverse pressure gradient on the lee side of the hill. Coelho and Pereira [32] studied the same problem computationally by using a non-orthogonal body fitted coordinate system with the Cartesian velocity components as the unknown variables. Standard $\mathcal{K}-\epsilon$ model of Launder and Spalding [14] and low Reynolds $\mathcal{K}-\epsilon$ model of Lam and Bremhorst [33] were used for turbulence closure.

The height of the hill is $H$. The domain extends to a distance of $15 \mathrm{H}$ upstream and $20 \mathrm{H}$ downstream of the hill. The height of the domain is $6.07 \mathrm{H}$ and the width is $7 \mathrm{H}$. Coelho and Pereira [32] conducted detailed numerical comparisons of 2D and 3D flow predictions. In their analysis, it was observed that despite the presence of the side walls, the flow remains two dimensional at the $z$ mid-plane. They concluded that "3D predictions using $\mathcal{K}-\epsilon$ eddy viscosity model at the central plane are virtually identical to 2 D simulations". Thus, in the present simulation, the end walls in the transverse $z$ direction are modeled as slip walls to make the flow two-dimensional. The top and bottom boundaries are viscous no-slip walls. A non-orthogonal body conforming grid of size $275 \times 70 \times 30$ is generated using the simple algebraic technique of Trans-Finite Interpolation (TFI) is used. TFI is a straightforward technique that can be used to control the grid density effectively in the near wall region. Figure 14a, 14b show the grids around the hill in the $x-y$ plane.

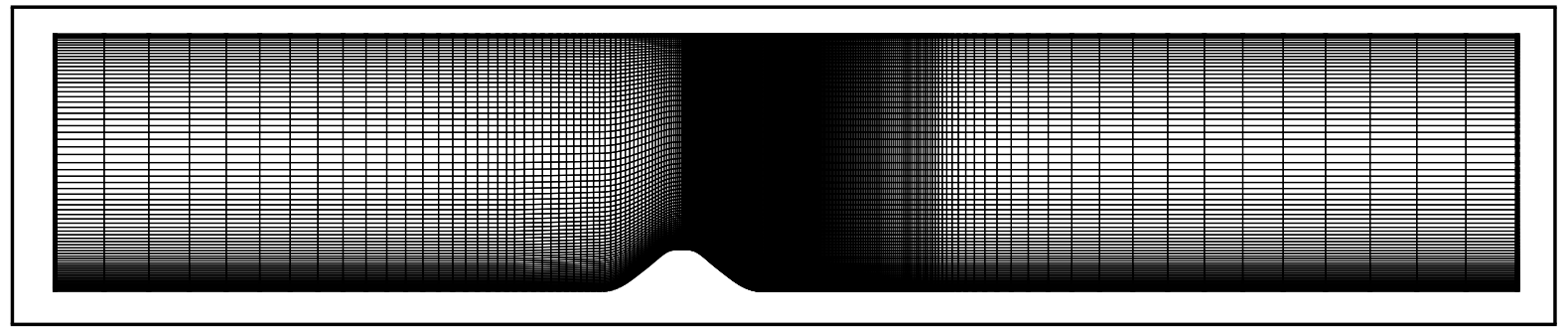

(a) Grid on the $x-y$ plane

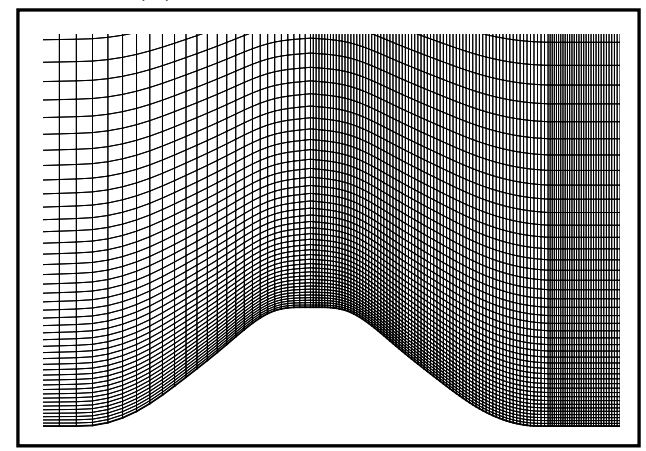

(b) Close up view of the grid near the hill

Fig. 14. Body conforming grid over the hill

The bulk inflow velocity is $U_{o}$. The flow is fully developed at the outlet and the conditions are determined through global mass conservation. The Reynolds number based on the hill height and the bulk inflow velocity is $R e=\rho U_{o} H / \mu=6 \times 10^{4}$. Figure 15 shows the contours of the mean streamline of the flow in the $z$ mid-plane. Separation of the flow occurs on the lee side of the hill, aft of the hill peak. The separation and reattachment points are found to be $x_{s} / H=0.47$ and $x_{r} / H=4.85$ respectively. It compares well with the experimentally observed values of $0.43,4.82[29]$ and the low Reynolds number turbulence model computational values of $0.43,4.64$ [32]. 


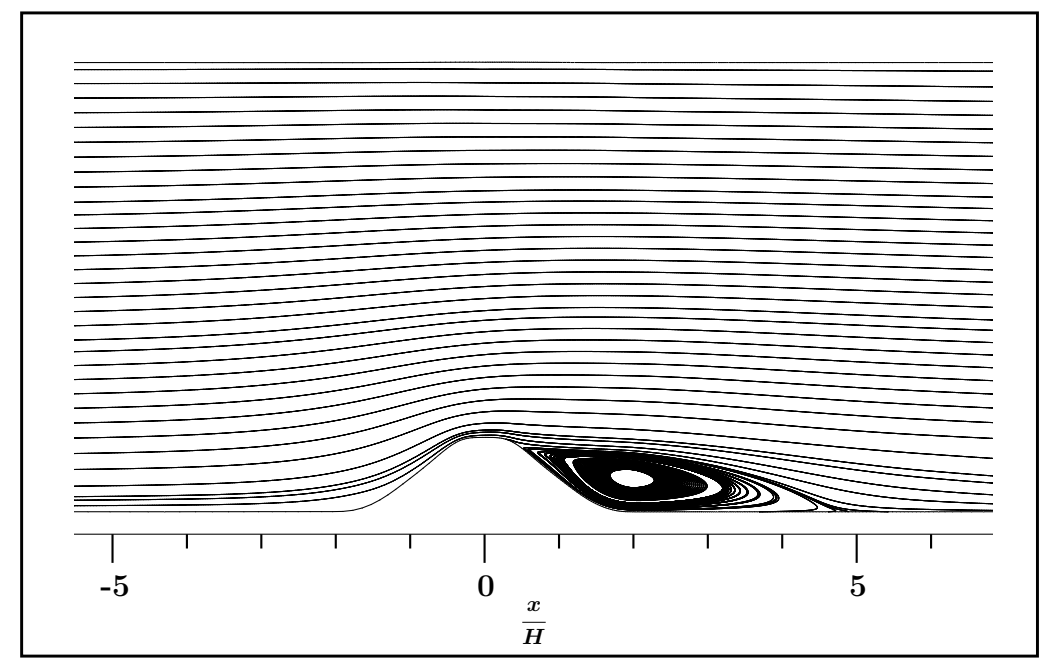

Fig. 15. Contours of the mean streamline for turbulent flow over a hill.

A very small separation region was observed near the front base of the hill as reported in Coelho and Pereira [32], which was not detected in the experiments. The very small separation occurs due to nonsmooth transition between the hill surface and the bottom wall. In the experiments of Almeida et al. [29], the separation point was estimated from the axial velocity measured at a distance of $1 \mathrm{~mm}$ from the wall and consequently the small separation region was not detected. Figures 16, 17 show the comparison of the predicted and the experimentally measured profiles of the mean velocity in the stream-wise and normal directions at the $z$ mid-plane. The plots correspond to the vertical profiles at 14 different horizontal locations ${ }^{1}$ of $x / H=-1.786,-0.714,0.0,1.071,1.786,2.5,3.214,4.286,4.786,5.357,6.607,8.036,10.714$ and 17.850 . The span-wise velocity was zero, confirming that the present simulation is essentially two-dimensional. The predicted mean flow shows overall good agreement with the experimentally observed values, specifically in the separated recirculation region. However, the present simulation shows a higher acceleration of the flow near the hill peak and under predicts the velocity in the boundary layer downstream of the reattachment point, a trend also observed in Coelho and Pereira [32].

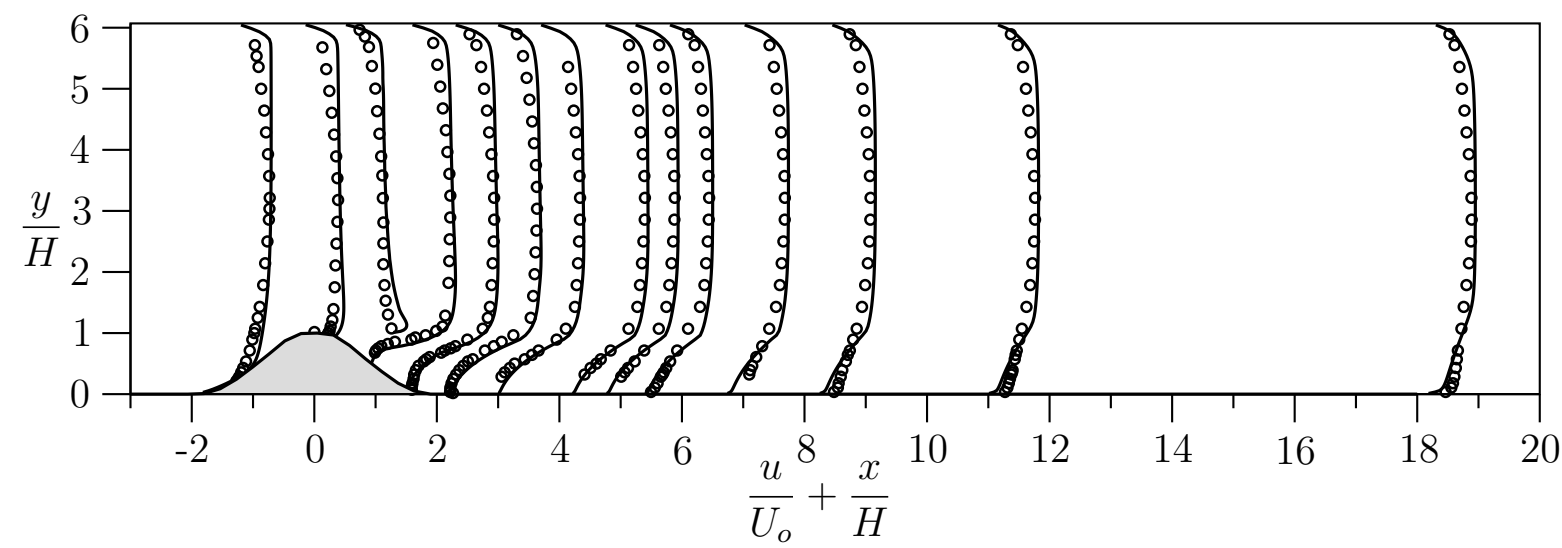

Fig. 16. Comparison of the $u$ velocity profiles for turbulent flow over a hill. Present simulation ( — ), Almeida et al. [29] experiments $(0)$

\footnotetext{
${ }^{1}$ In [29], the first two $x / H$ stations are reported to be located at $-1.964,-0.724$. However numerous sources including [31, $32,34]$ indicate that the first two stations are located at $-1.786,-0.714$. The present simulation also confirms the same, based on the velocity profile comparisons.
} 


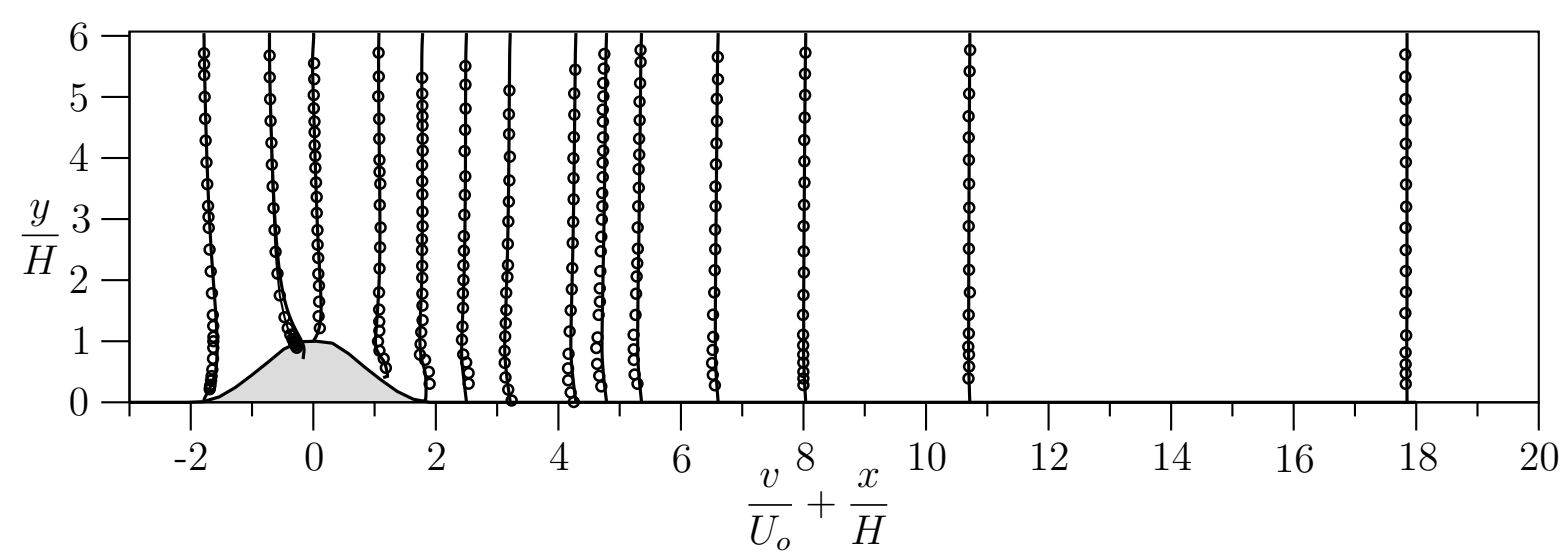

Fig. 17. Comparison of the $v$ velocity profiles for turbulent flow over a hill. Present simulation ( — ), Almeida et al. [29] experiments $(\circ)$

\section{Conclusion}

The governing conservation equations for a Reynolds averaged turbulent flow have been redeveloped in a new mixed contravariant-covariant basis for a general curvilinear non-orthogonal body fitted coordinate system. The mixed form representation of the momentum equations in non-orthogonal coordinates closely resemble orthogonal equations and has the advantages of both the covariant and contravariant formulations. The new mixed form of the conservation equations has the advantage of the contravariant formulation in that the convective flux at a face is a single term similar to the orthogonal coordinates and in addition retains the diagonally dominant pressure characteristics of the covariant form of the equations. The advantages and disadvantages of the Navier-Stokes equations based on different velocity formulations are summarized in the Table 5.

Table 5. Comparison of the Navier-Stokes equations based on different velocity formulations

\begin{tabular}{|c|c|c|}
\hline Formulation & Advantages & Disadvantages \\
\hline Cartesian & $\begin{array}{c}\text { Simple equations without secondary } \\
\text { source terms }\end{array}$ & $\begin{array}{l}\text { Complex } 9 / 21 \text { point pressure equation } \\
\text { stencil lacking diagonal dominance }\end{array}$ \\
\hline Contravariant & $\begin{array}{l}\text { Convective flux calculated with single } \\
\text { velocity component }\end{array}$ & $\begin{array}{l}\text { Complex } 9 / 21 \text { point pressure equation } \\
\text { stencil lacking diagonal dominance and } \\
\text { complex secondary source terms }\end{array}$ \\
\hline Covariant & $\begin{array}{c}\text { Diagonally dominant, simple } 5 / 7 \text { point } \\
\text { pressure equation stencil }\end{array}$ & $\begin{array}{l}\text { Interpolation of non-directional velocities } \\
\text { for computing convective fluxes and } \\
\text { complex secondary source terms }\end{array}$ \\
\hline $\begin{array}{l}\text { Mixed } \\
\text { contravariant- } \\
\text { covariant }\end{array}$ & $\begin{array}{l}\text { Diagonally dominant, simple } 5 / 7 \text { point } \\
\text { pressure equation stencil. Convective flux } \\
\text { calculated with single velocity component }\end{array}$ & Complex secondary source terms \\
\hline
\end{tabular}

The proposed formulation is the basis of a three-dimensional RANS solver. Laminar flow in a lid driven skewed cavity, $90^{\circ}$ L-bend and turbulent flow over a hill are tested with curvilinear grids. Good agreement is observed between the present simulations and numerical/experimental results from the literature.

\section{Acknowledgments}

Authors would like to thank the two anonymous reviewers whose insightful comments helped in improving this article. We would like to express gratitude to the National Science Foundation and Iowa - EPSCOR. 
This material is based upon work primarily supported by the National Science Foundation under NSF Grant Number EPS-1101284. Any opinions, findings, and conclusions or recommendations expressed in this material are those of the authors and do not necessarily reflect the views of the National Science Foundation. We would also like to thank the Department of Aerospace Engineering, Iowa State University for providing partial financial support.

\section{Appendix A. Divergence of a second rank mixed component tensor}

The mathematical operation of divergence, which reduces the rank of a tensor by one, is given by

$$
\nabla \cdot()=\frac{\partial()}{\partial x^{k}} \cdot e^{k}
$$

Expressing a second rank tensor using mixed components in a contravariant-covariant basis $(\tilde{\boldsymbol{T}}=$ $\left.T_{i}^{j} \boldsymbol{e}^{\boldsymbol{i}} \boldsymbol{e}_{\boldsymbol{j}}\right)$, the divergence of a tensor is

$$
\nabla \cdot \tilde{\boldsymbol{T}}=\frac{\partial}{\partial x^{k}}\left(T_{i}^{j} \boldsymbol{e}^{i} \boldsymbol{e}_{j}\right) \cdot \boldsymbol{e}^{\boldsymbol{k}}
$$

Expanding the above equation

$$
\boldsymbol{\nabla} \cdot \tilde{\boldsymbol{T}}=\left[\frac{\partial T_{i}^{j}}{\partial x^{k}} \boldsymbol{e}^{i} \boldsymbol{e}_{\boldsymbol{j}}+T_{i}^{j} \frac{\partial \boldsymbol{e}^{i}}{\partial x^{k}} \boldsymbol{e}_{\boldsymbol{j}}+T_{i}^{j} \boldsymbol{e}^{i} \frac{\partial \boldsymbol{e}_{j}}{\partial x^{k}}\right] \cdot \boldsymbol{e}^{\boldsymbol{k}}
$$

The Christoffel symbols of the second kind are given by

$$
\Gamma_{i j}^{k}=\frac{\partial \boldsymbol{e}_{i}}{\partial x^{j}} \cdot \boldsymbol{e}^{\boldsymbol{k}}=-\frac{\partial \boldsymbol{e}^{k}}{\partial x^{j}} \cdot \boldsymbol{e}_{\boldsymbol{i}}
$$

Equation (A.4) and the mutual reciprocity of bases $\boldsymbol{e}_{\boldsymbol{i}} \cdot \boldsymbol{e}^{j}=\delta_{i}^{j}$ implies

$$
\Gamma_{i j}^{k} e^{i}=-\frac{\partial e^{k}}{\partial x^{j}}
$$

Expanding one term at a time in Eq. (A.3)

1. Using mutual reciprocity of the bases, $\boldsymbol{e}_{j} \cdot \boldsymbol{e}^{k}=\delta_{j}^{k}$

2. Using Eq. (A.5)

$$
\frac{\partial T_{i}^{j}}{\partial x^{k}} e^{i} e_{j} \cdot e^{k}=\frac{\partial T_{i}^{j}}{\partial x^{k}} e^{i} \delta_{j}^{k}=\frac{\partial T_{i}^{j}}{\partial x^{j}} e^{i}
$$

$$
T_{i}^{j} \frac{\partial \boldsymbol{e}^{i}}{\partial x^{k}} \boldsymbol{e}_{\boldsymbol{j}} \cdot \boldsymbol{e}^{\boldsymbol{k}}=T_{i}^{j} \frac{\partial \boldsymbol{e}^{i}}{\partial x^{k}} \delta_{j}^{k}=T_{i}^{j} \frac{\partial \boldsymbol{e}^{i}}{\partial x^{j}}=T_{k}^{j} \frac{\partial \boldsymbol{e}^{k}}{\partial x^{j}}=-T_{k}^{j} \Gamma_{i j}^{k} \boldsymbol{e}^{i}
$$

3. Using Eq. (A.4) and the specific property of the Christoffel symbols of the second kind with repeated index [12], $\Gamma_{j k}^{k}=\frac{1}{2 g} \frac{\partial g}{\partial x^{j}}$

$$
T_{i}^{j} \boldsymbol{e}^{i} \frac{\partial \boldsymbol{e}_{j}}{\partial x^{k}} \cdot \boldsymbol{e}^{\boldsymbol{k}}=T_{i}^{j} \boldsymbol{e}^{i} \Gamma_{j k}^{k}=T_{i}^{j} \frac{1}{2 g} \frac{\partial g}{\partial x^{j}} \boldsymbol{e}^{i}
$$

Combining the three terms of Eqs. (A.6) - (A.8),

$$
\boldsymbol{\nabla} \cdot \tilde{\boldsymbol{T}}=\left[\frac{\partial T_{i}^{j}}{\partial x^{j}}-T_{k}^{j} \Gamma_{i j}^{k}+T_{i}^{j} \frac{1}{2 g} \frac{\partial g}{\partial x^{j}}\right] \boldsymbol{e}^{i}
$$

Combining the first and the last term yields the divergence of a second rank mixed component tensor.

$$
\boldsymbol{\nabla} \cdot \tilde{\boldsymbol{T}}=\left[\frac{1}{\sqrt{g}} \frac{\partial}{\partial x^{j}}\left(\sqrt{g} T_{i}^{j}\right)-T_{k}^{j} \Gamma_{i j}^{k}\right] \boldsymbol{e}^{i}
$$




\section{Appendix B. Gradient of a vector}

The mathematical gradient operator is given by

$$
\nabla()=\frac{\partial()}{\partial x^{k}} e^{k}
$$

Expressing a vector using covariant components in contravariant basis $\left(\boldsymbol{V}=V_{i} e^{i}\right)$, the gradient of a vector is

$$
\nabla \boldsymbol{V}=\frac{\partial}{\partial x^{k}}\left(V_{i} e^{i}\right) e^{k}
$$

Expanding the above equation

$$
\boldsymbol{\nabla} \boldsymbol{V}=\left[\frac{\partial V_{i}}{\partial x^{k}} \boldsymbol{e}^{i}+V_{i} \frac{\partial \boldsymbol{e}^{i}}{\partial x^{k}}\right] \boldsymbol{e}^{\boldsymbol{k}}
$$

Replacing the dummy index ' $i$ ' with ' $l$ ' in the second term

$$
\boldsymbol{\nabla} \boldsymbol{V}=\left[\frac{\partial V_{i}}{\partial x^{k}} \boldsymbol{e}^{i}+V_{l} \frac{\partial \boldsymbol{e}^{l}}{\partial x^{k}}\right] \boldsymbol{e}^{\boldsymbol{k}}
$$

Using Eq. (A.5), Eq. (B.4) can be written as

$$
\nabla \boldsymbol{V}=\left[\frac{\partial V_{i}}{\partial x^{k}} e^{i}-V_{l} \Gamma_{i k}^{l} e^{i}\right] e^{k}
$$

The gradient of a vector, can thus be expressed in pure contravariant basis as

$$
\nabla \boldsymbol{V}=\left[\frac{\partial V_{i}}{\partial x^{k}}-V_{l} \Gamma_{i k}^{l}\right] e^{i} e^{k}
$$

To get the mixed form representation of the above tensor, the basis of the second vector can be changed from contravariant to covariant.

$$
\boldsymbol{\nabla} \boldsymbol{V}=\left[\frac{\partial V_{i}}{\partial x^{k}}-V_{l} \Gamma_{i k}^{l}\right] \boldsymbol{e}^{i} g^{k j} \boldsymbol{e}_{\boldsymbol{j}}
$$

Making the substitution of the non-physical components with their corresponding physical counterparts, leads to the final required form.

$$
\boldsymbol{\nabla} \boldsymbol{V}=h^{i} h_{j}\left[\frac{\partial}{\partial x^{k}}\left(\frac{v_{i}}{h^{i}}\right)-\frac{v_{l}}{h^{l}} \Gamma_{i k}^{l}\right] g^{k j} \hat{\boldsymbol{e}}^{i} \hat{\boldsymbol{e}}_{\boldsymbol{j}}
$$

\section{Appendix C. Secondary diffusive fluxes}

The secondary diffusive fluxes $\psi_{1}^{1}, \psi_{1}^{2}$ and $\psi_{1}^{3}$ in Eq. (41) are given by

$$
\begin{gathered}
\psi_{1}^{1}=-2\left(\mu+\mu_{t}\right) h^{1} h_{1}\left\{g^{11}\left[\frac{1}{2} \frac{\partial}{\partial x^{1}}\left(\frac{v_{1}}{h^{1}}\right)-\frac{1}{2} \frac{v_{1}}{\left(h^{1}\right)^{2}} \frac{\partial h^{1}}{\partial x^{1}}-\frac{v_{1}}{h^{1}} \Gamma_{11}^{1}-\frac{v_{2}}{h^{2}} \Gamma_{11}^{2}-\frac{v_{3}}{h^{3}} \Gamma_{11}^{3}\right]\right. \\
+g^{21}\left[\frac{\partial}{\partial x^{2}}\left(\frac{v_{1}}{h^{1}}\right)-\frac{v_{1}}{h^{1}} \Gamma_{12}^{1}-\frac{v_{2}}{h^{2}} \Gamma_{12}^{2}-\frac{v_{3}}{h^{3}} \Gamma_{12}^{3}\right] \\
\left.+g^{31}\left[\frac{\partial}{\partial x^{3}}\left(\frac{v_{1}}{h^{1}}\right)-\frac{v_{1}}{h^{1}} \Gamma_{13}^{1}-\frac{v_{2}}{h^{2}} \Gamma_{13}^{2}-\frac{v_{3}}{h^{3}} \Gamma_{13}^{3}\right]\right\}
\end{gathered}
$$




$$
\begin{aligned}
& \psi_{1}^{2}=-\left(\mu+\mu_{t}\right)\{ h^{1} h_{2} g^{12}\left[\frac{\partial}{\partial x^{1}}\left(\frac{v_{1}}{h^{1}}\right)-\frac{v_{1}}{h^{1}} \Gamma_{11}^{1}-\frac{v_{2}}{h^{2}} \Gamma_{11}^{2}-\frac{v_{3}}{h^{3}} \Gamma_{11}^{3}\right] \\
&+h^{1} h_{2} g^{22}\left[-\frac{v_{1}}{\left.h^{1}\right)^{2}} \frac{\partial h^{1}}{\partial x^{2}}-\frac{v_{1}}{h^{1}} \Gamma_{12}^{1}-\frac{v_{2}}{h^{2}} \Gamma_{12}^{2}-\frac{v_{3}}{h^{3}} \Gamma_{12}^{3}\right] \\
&+h^{1} h_{2} g^{32}\left[\frac{\partial}{\partial x^{3}}\left(\frac{v_{1}}{h^{1}}\right)-\frac{v_{1}}{h^{1}} \Gamma_{13}^{1}-\frac{v_{2}}{h^{2}} \Gamma_{13}^{2}-\frac{v_{3}}{h^{3}} \Gamma_{13}^{3}\right] \\
&+h^{2} h_{1} g^{11}\left[\frac{\partial}{\partial x^{1}}\left(\frac{v_{2}}{h^{2}}\right)-\frac{v_{1}}{h^{1}} \Gamma_{21}^{1}-\frac{v_{2}}{h^{2}} \Gamma_{21}^{2}-\frac{v_{3}}{h^{3}} \Gamma_{21}^{3}\right] \\
&+h^{2} h_{1} g^{21}\left[\frac{\partial}{\partial x^{2}}\left(\frac{v_{2}}{h^{2}}\right)-\frac{v_{1}}{h^{1}} \Gamma_{22}^{1}-\frac{v_{2}}{h^{2}} \Gamma_{22}^{2}-\frac{v_{3}}{h^{3}} \Gamma_{22}^{3}\right] \\
&\left.+h^{2} h_{1} g^{31}\left[\frac{\partial}{\partial x^{3}}\left(\frac{v_{2}}{h^{2}}\right)-\frac{v_{1}}{h^{1}} \Gamma_{23}^{1}-\frac{v_{2}}{h^{2}} \Gamma_{23}^{2}-\frac{v_{3}}{h^{3}} \Gamma_{23}^{3}\right]\right\} \\
& \psi_{1}^{3}=-\left(\mu+\mu_{t}\right)\left\{h^{1} h_{3} g^{13}\left[\frac{\partial}{\partial x^{1}}\left(\frac{v_{1}}{h^{1}}\right)-\frac{v_{1}}{h^{1}} \Gamma_{11}^{1}-\frac{v_{2}}{h^{2}} \Gamma_{11}^{2}-\frac{v_{3}}{h^{3}} \Gamma_{11}^{3}\right]\right. \\
&+h^{1} h_{3} g^{23}\left[\frac{\partial}{\partial x^{2}}\left(\frac{v_{1}}{h^{1}}\right)-\frac{v_{1}}{h^{1}} \Gamma_{12}^{1}-\frac{v_{2}}{h^{2}} \Gamma_{12}^{2}-\frac{v_{3}}{h^{3}} \Gamma_{12}^{3}\right] \\
&+h^{1} h_{3} g^{33}\left[-\frac{v_{1}}{\left(h^{1}\right)^{2}} \frac{\partial h^{1}}{\partial x^{3}}-\frac{v_{1}}{h^{1}} \Gamma_{13}^{1}-\frac{v_{2}}{h^{2}} \Gamma_{13}^{2}-\frac{v_{3}}{h^{3}} \Gamma_{13}^{3}\right] \\
&+h^{3} h_{1} g^{11}\left[\frac{\partial}{\partial x^{1}}\left(\frac{v_{3}}{h^{3}}\right)-\frac{v_{1}}{h^{1}} \Gamma_{31}^{1}-\frac{v_{2}}{h^{2}} \Gamma_{31}^{2}-\frac{v_{3}}{h^{3}} \Gamma_{31}^{3}\right] \\
&+h^{3} h_{1} g^{21}\left[\frac{\partial}{\partial x^{2}}\left(\frac{v_{3}}{h^{3}}\right)-\frac{v_{1}}{h^{1}} \Gamma_{32}^{1}-\frac{v_{2}}{h^{2}} \Gamma_{32}^{2}-\frac{v_{3}}{h^{3}} \Gamma_{32}^{3}\right] \\
&\left.+h^{3} h_{1} g^{31}\left[\frac{\partial}{\partial x^{3}}\left(\frac{v_{3}}{h^{3}}\right)-\frac{v_{1}}{h^{1}} \Gamma_{33}^{1}-\frac{v_{2}}{h^{2}} \Gamma_{33}^{2}-\frac{v_{3}}{h^{3}} \Gamma_{33}^{3}\right]\right\} \\
&
\end{aligned}
$$

\section{Appendix D. Influence of multi-directional pressure gradient on the diagonal dominance of pressure equation.}

The mass and momentum conservation equations using contravariant velocity can be obtained in a manner similar to Eqs. (26), (28).

$$
\begin{aligned}
\frac{\partial \rho}{\partial t}+\frac{1}{\sqrt{g}} \frac{\partial}{\partial x^{i}}\left(\frac{\rho \sqrt{g} v^{i}}{h_{i}}\right) & =0 \\
\frac{\partial \rho v^{i}}{\partial t}+\frac{1}{\sqrt{g}} \frac{\partial}{\partial x^{j}}\left(\frac{\sqrt{g} t^{i j}}{h_{j}}\right) & =-h_{i} g^{i j} \frac{\partial p}{\partial x^{j}}+s^{i}
\end{aligned}
$$

where $s^{i}$ contains all the secondary source terms and any additional terms like turbulent kinetic energy. The above momentum equation contains multi-directional pressure gradient because of the non-orthogonal metric $g^{i j}$. For the sake of brevity, the pressure equation is obtained in two dimensions here. For an incompressible flow, the mass Eq. (D.1) can be expanded as,

$$
\frac{1}{\sqrt{g}} \frac{\partial}{\partial x^{1}}\left(\frac{\rho \sqrt{g} v^{1}}{h_{1}}\right)+\frac{1}{\sqrt{g}} \frac{\partial}{\partial x^{2}}\left(\frac{\rho \sqrt{g} v^{2}}{h_{2}}\right)=0
$$

The above equation can be integrated over a control volume $\Delta \forall=\sqrt{g} \Delta x^{1} \Delta x^{2}$ and discretized similar to Eq. (58).

$$
\left(\rho A_{e} v_{e}^{1}\right)-\left(\rho A_{w} v_{w}^{1}\right)+\left(\rho A_{n} v_{n}^{2}\right)-\left(\rho A_{s} v_{s}^{2}\right)=0
$$


The area terms are $A_{e}=\left(\sqrt{g} \Delta x^{2} / h_{1}\right)_{e}, A_{w}=\left(\sqrt{g} \Delta x^{2} / h_{1}\right)_{w}, A_{n}=\left(\sqrt{g} \Delta x^{1} / h_{2}\right)_{n}, A_{s}=\left(\sqrt{g} \Delta x^{1} / h_{2}\right)_{s}$.

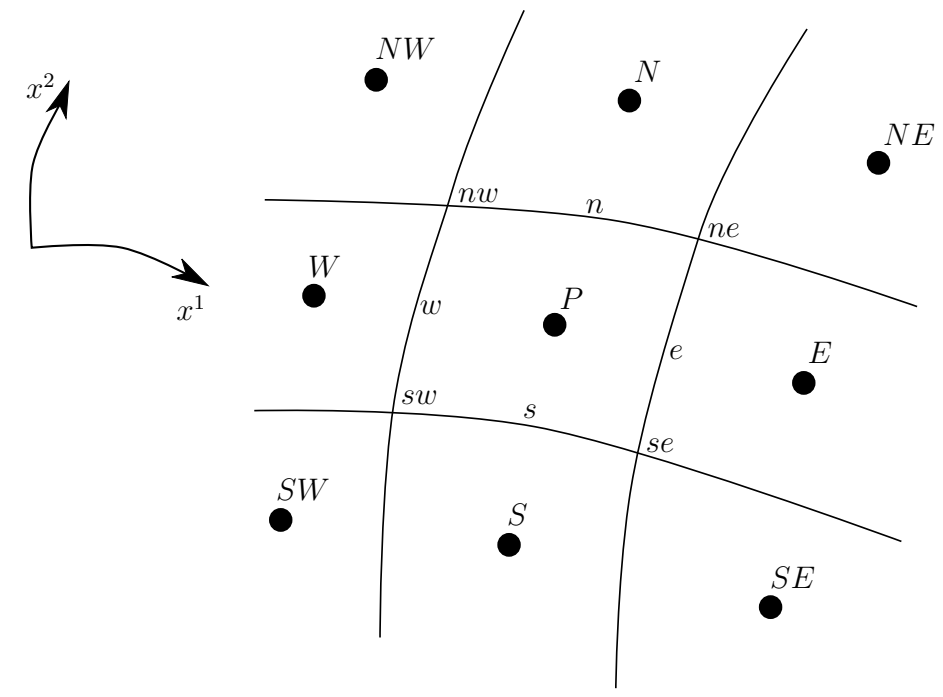

Fig. 18. 9 point stencil for the pressure equation.

Using the stencil shown in Fig. 18, the equation for $v^{1}$ velocity at $e$ face can be obtained in a manner similar to Eq. (59) by integrating and discretizing Eq. (D.2).

$$
a_{e} v_{e}^{1}=\sum a_{n b} v_{n b}^{1}+b_{e}-\left(h_{1} g^{11} \sqrt{g} \Delta x^{2}\right)_{e}\left(p_{E}-p_{P}\right)-\left(h_{1} g^{12} \sqrt{g} \Delta x^{1}\right)_{P E}\left(p_{n e}-p_{s e}\right)
$$

Rearranging,

$$
v_{e}^{1}=\hat{v}_{e}^{1}+d_{e}\left(p_{P}-p_{E}\right)+e_{P E}^{\prime}\left(p_{s e}-p_{n e}\right)
$$

where

$$
\hat{v}_{e}^{1}=\left(\Sigma a_{n b} v_{n b}^{1}+b_{e}\right) / a_{e} \quad d_{e}=\left(h_{1} g^{11} \sqrt{g} \Delta x^{2}\right)_{e} / a_{e} \quad e_{P E}^{\prime}=\left(h_{1} g^{12} \sqrt{g} \Delta x^{1}\right)_{P E} / a_{e}
$$

It may be noted that $d_{e}$ is always positive, while the sign of $e_{P E}^{\prime}$ depends on the grid non-orthogonal metric $g^{12}$. If a simple bi-linear scheme is used to interpolate $p_{s e}, p_{n e}$ on a uniform grid such that $p_{s e}=$ $0.25\left(p_{S}+p_{S E}+p_{P}+p_{E}\right), p_{n e}=0.25\left(p_{P}+p_{E}+p_{N}+p_{N E}\right)$, then

$$
v_{e}^{1}=\hat{v}_{e}^{1}+d_{e}\left(p_{P}-p_{E}\right)+e_{P E}\left(p_{S}+p_{S E}-p_{N}-p_{N E}\right)
$$

The factor 0.25 can be absorbed into $e_{P E}^{\prime}$ and denoted as $e_{P E}$. Similar discrete equations can be obtained for the velocities at the other three faces.

$$
\begin{aligned}
v_{w}^{1} & =\hat{v}_{w}^{1}+d_{w}\left(p_{W}-p_{P}\right)+e_{W P}\left(p_{S W}+p_{S}-p_{N W}-p_{N}\right) \\
v_{n}^{2} & =\hat{v}_{n}^{2}+d_{n}\left(p_{P}-p_{N}\right)+e_{P N}\left(p_{W}+p_{N W}-p_{E}-p_{N E}\right) \\
v_{s}^{2} & =\hat{v}_{s}^{2}+d_{s}\left(p_{S}-p_{P}\right)+e_{S P}\left(p_{S W}+p_{W}-p_{S E}-p_{E}\right)
\end{aligned}
$$

Substituting $v_{e}^{1}, v_{w}^{1}, v_{n}^{2}, v_{s}^{2}$ into Eq. (D.4) and rearranging,

$$
a_{P} p_{P}=a_{E} p_{E}+a_{W} p_{W}+a_{N} p_{N}+a_{S} p_{S}+a_{S E} p_{S E}+a_{N E} p_{N E}+a_{S W} p_{S W}+a_{N W} p_{N W}+\hat{b}_{P}
$$


where

$$
\begin{aligned}
a_{E} & =\left(\rho A_{e} d_{e}\right)+\left(\rho A_{n} e_{P N}\right)-\left(\rho A_{s} e_{S P}\right) \\
a_{W} & =\left(\rho A_{w} d_{w}\right)-\left(\rho A_{n} e_{P N}\right)+\left(\rho A_{s} e_{S P}\right) \\
a_{N} & =\left(\rho A_{n} d_{n}\right)+\left(\rho A_{e} e_{P E}\right)-\left(\rho A_{w} e_{W P}\right) \\
a_{S} & =\left(\rho A_{s} d_{s}\right)-\left(\rho A_{e} e_{P E}\right)+\left(\rho A_{w} e_{W P}\right) \\
a_{S E} & =-\left(\rho A_{s} e_{S P}\right)-\left(\rho A_{e} e_{P E}\right) \\
a_{N E} & =\left(\rho A_{n} e_{P N}\right)+\left(\rho A_{e} e_{P E}\right) \\
a_{S W} & =\left(\rho A_{s} e_{S P}\right)+\left(\rho A_{w} e_{W P}\right) \\
a_{N W} & =-\left(\rho A_{n} e_{P N}\right)-\left(\rho A_{w} e_{W P}\right) \\
a_{P} & =a_{E}+a_{W}+a_{N}+a_{S}+a_{S E}+a_{N E}+a_{S W}+a_{N W} \\
\hat{b}_{P} & =\left(\rho A_{w} \hat{v}_{w}^{1}\right)-\left(\rho A_{e} \hat{v}_{e}^{1}\right)+\left(\rho A_{s} \hat{v}_{s}^{2}\right)-\left(\rho A_{n} \hat{v}_{n}^{2}\right)
\end{aligned}
$$

Thus, in 2D, the pressure equation has a nine point stencil. The neighbor coefficients $a_{n b}$ may be positive or negative depending on local grid non-orthogonality. It is possible for the central coefficient $a_{P}$ to be lower than $\sum\left|a_{n b}\right|$ and diagonal dominance is no longer guaranteed.

\section{References}

[1] CM Rhie and WL Chow. Numerical study of the turbulent flow past an airfoil with trailing edge separation. $A I A A$ journal, 21(11):1525-1532, 1983.

[2] FS Lien and MA Leschziner. A general non-orthogonal collocated finite volume algorithm for turbulent flow at all speeds incorporating second-moment turbulence-transport closure, part 1: Computational implementation. Computer methods in applied mechanics and engineering, 114(1):123-148, 1994.

[3] Wei Shyy, SS Tong, and SM Correa. Numerical recirculating flow calculation using a body-fitted coordinate system. Numerical Heat Transfer, 8(1):99-113, 1985.

[4] M Peric. Analysis of pressure-velocity coupling on nonorthogonal grids. Numerical Heat Transfer, 17:63-82, 1990.

[5] I Demirdzic, AD Gosman, RI Issa, and M Peric. A calculation procedure for turbulent flow in complex geometries. Computers \&J fluids, 15(3):251-273, 1987.

[6] HQ Yang, KT Yang, and JR Lloyd. A control volume finite difference method for buoyant flow in three-dimensional curvilinear non-orthogonal co-ordinates. International Journal for Numerical Methods in Fluids, 10(2):199-211, 1990.

[7] J Graef, R Shivaji, B Soni, and J Zhu. Governing equations of fluid mechanics in physical curvilinear coordinate system. In Differential Equations and Computational Simulations, Conference, volume 1, 1997.

[8] MC Sharatchandra and DL Rhode. New. strongly conservative finite-volume formulation for fluid flows in irregular geometries using contravariant velocity components: Part 1. theory. Numerical Heat Transfer, 26(1):39-52, 1994.

[9] KC Karki and SV Patankar. Calculation procedure for viscous incompressible flows in complex geometries. Numerical Heat Transfer, Part A: Applications, 14(3):295-307, 1988.

[10] P Tamamidis and DN Assanis. Prediction of three-dimensional steady incompressible flows using body-fitted coordinates. Journal of Fluids Engineering, 115(3):457-462, 1993.

[11] HQ Yang, Sami D Habchi, and Andrzej J Przekwas. General strong conservation formulation of navier-stokes equations in nonorthogonal curvilinear coordinates. AIAA journal, 32(5):936-941, 1994.

[12] Zahir UA Warsi. Fluid dynamics: theoretical and computational approaches. CRC press, 2005.

[13] Suhas Patankar. Numerical heat transfer and fluid flow. CRC Press, 1980.

[14] Brian Edward Launder and DB Spalding. The numerical computation of turbulent flows. Computer methods in applied mechanics and engineering, 3(2):269-289, 1974.

[15] M Kato. The modeling of turbulent flow around stationary and vibrating square cylinders. In Ninth Symposium on Turbulent Shear Flows, 1993, 1993.

[16] Douglas L Sondak and Richard H Pletcher. Application of wall functions to generalized nonorthogonal curvilinear coordinate systems. AIAA journal, 33(1):33-41, 1995.

[17] I Demirdžić, ̌̌ Lilek, and M Perić. Fluid flow and heat transfer test problems for non-orthogonal grids: Bench-mark solutions. International Journal for Numerical Methods in Fluids, 15(3):329-354, 1992.

[18] CW Oosterlee, P Wesseling, A Segal, and E Brakkee. Benchmark solutions for the incompressible navier-stokes equations in general co-ordinates on staggered grids. International Journal for Numerical Methods in Fluids, 17(4):301-321, 1993.

[19] D Ghosh Roychowdhury, Sarit Kumar Das, and T Sundararajan. An efficient solution method for incompressible n-s equations using non-orthogonal collocated grid. International Journal for numerical methods in engineering, 45(6):741$763,1999$.

[20] J Rafael Pacheco and Robert E Peck. Nonstaggered boundary-fitted coordinate method for free surface flows. Numerical Heat Transfer: Part B: Fundamentals, 37(3):267-291, 2000. 
[21] PG Tucker and Z Pan. A cartesian cut cell method for incompressible viscous flow. Applied Mathematical Modelling, 24(8):591-606, 2000.

[22] Y Wang and S Komori. On the improvement of the simple-like method for flows with complex geometry. Heat and mass transfer, 36(1):71-78, 2000.

[23] $\mathrm{H} \mathrm{Xu}$ and $\mathrm{C}$ Zhang. Numerical calculations of laminar flows using contravariant velocity fluxes. Computers 8 fluids, 29(2):149-177, 2000.

[24] Rune Teigland and Inge K Eliassen. A multiblock/multilevel mesh refinement procedure for cfd computations. International journal for numerical methods in fluids, 36(5):519-538, 2001.

[25] A Shklyar and A Arbel. Numerical method for calculation of the incompressible flow in general curvilinear co-ordinates with double staggered grid. International journal for numerical methods in fluids, 41(12):1273-1294, 2003.

[26] Ercan Erturk and Bahtiyar Dursun. Numerical solutions of 2-d steady incompressible flow in a driven skewed cavity. ZAMM-Journal of Applied Mathematics and Mechanics/Zeitschrift für Angewandte Mathematik und Mechanik, 87(5):377-392, 2007.

[27] Moshe Rosenfeld, Dochan Kwak, and Marcel Vinokur. A fractional step solution method for the unsteady incompressible navier-stokes equations in generalized coordinate systems. Journal of Computational Physics, 94(1):102-137, 1991.

[28] JAC Humphrey, AMK Taylor, and JH Whitelaw. Laminar flow in a square duct of strong curvature. Journal of Fluid Mechanics, 83(03):509-527, 1977.

[29] GP Almeida, DFG Durao, and MV Heitor. Wake flows behind two-dimensional model hills. Experimental Thermal and Fluid Science, 7(1):87-101, 1993.

[30] JCR Hunt and WH Snyder. Experiments on stably and neutrally stratified flow over a model three-dimensional hill. Journal of Fluid Mechanics, 96(04):671-704, 1980.

[31] ERCOFTAC. European Research Community on Flow, Turbulence and Combustion Database. [http://cfd.mace.manchester.ac.uk./ercoftac/], 2014.

[32] PJ Coelho and JCF Pereira. Finite volume computation of the turbulent flow over a hill employing 2 d or $3 \mathrm{~d}$ non-orthogonal collocated grid systems. International journal for numerical methods in fluids, 14(4):423-441, 1992.

[33] CKG Lam and $\mathrm{K}$ Bremhorst. A modified form of the $\mathrm{k}-\varepsilon$ model for predicting wall turbulence. Journal of Fluids Engineering, 103(3):456-460, 1981.

[34] W Rodi, JC Bonnin, and T Buchal. Ercoftac workshop on data bases and testing of calculation methods for turbulent flows. Technical report, University of Karsruhe, 1995. 